\title{
EQUATIONS FOR SECANT VARIETIES OF VERONESE AND OTHER VARIETIES
}

\author{
J.M. LANDSBERG AND GIORGIO OTTAVIANI
}

\begin{abstract}
New classes of modules of equations for secant varieties of Veronese varieties are defined using representation theory and geometry. Some old modules of equations (catalecticant minors) are revisited to determine when they are sufficient to give scheme-theoretic defining equations. An algorithm to decompose a general ternary quintic as the sum of seven fifth powers is given as an illustration of our methods. Our new equations and results about them are put into a larger context by introducing vector bundle techniques for finding equations of secant varieties in general. We include a few homogeneous examples of this method.
\end{abstract}

\section{INTRODUCTION}

1.1. Statement of problem and main results. Let $S^{d} \mathbb{C}^{n+1}=S^{d} V$ denote the space of homogeneous polynomials of degree $d$ in $n+1$ variables, equivalently the space of symmetric $d$-way tensors over $\mathbb{C}^{n+1}$. It is an important problem for complexity theory, signal processing, algebraic statistics, and many other areas (see e.g., [7, 11, 41, 27]) to find tests for the border rank of a given tensor. Geometrically, in the symmetric case, this amounts to finding set-theoretic defining equations for the secant varieties of the Veronese variety $v_{d}(\mathbb{P} V) \subset \mathbb{P} V$, the variety of rank one symmetric tensors.

For an algebraic variety $X \subset \mathbb{P} W$, the $r$-th secant variety $\sigma_{r}(X)$ is defined by

$$
\sigma_{r}(X)=\overline{\bigcup_{x_{1}, \ldots, x_{r} \in X} \mathbb{P}\left\langle x_{1}, \ldots, x_{r}\right\rangle} \subset \mathbb{P} W
$$

where $\left\langle x_{1}, \ldots, x_{r}\right\rangle \subset W$ denotes the linear span of the points $x_{1}, \ldots, x_{r}$ and the overline denotes Zariski closure. When $X=v_{d}(\mathbb{P} V), \sigma_{r}(X)$ is the Zariski closure of the set of polynomials that are the sum of $r d$-th powers.

When $d=2, S^{2} V$ may be thought of as the space of $(n+1) \times(n+1)$ symmetric matrices via the inclusion $S^{2} V \subset V \otimes V$ and the equations for $\sigma_{r}\left(v_{2}(\mathbb{P} V)\right)$ are just the size $r+1$ minors (these equations even generate the ideal). The first equations found for secant varieties of higher Veronese varieties were obtained by imitating this construction, considering the inclusions $S^{d} V \subset S^{a} V \otimes S^{d-a} V$, where $1 \leq a \leq\left\lfloor\frac{d}{2}\right\rfloor$ : Given $\phi \in S^{d} V$, one considers the corresponding linear map $\phi_{a, d-a}: S^{a} V^{*} \rightarrow S^{d-a} V$ and if $\phi \in \sigma_{r}\left(v_{d}(\mathbb{P} V)\right)$, then $\operatorname{rank}\left(\phi_{a, d-a}\right) \leq r$, see $\S 2.2$. Such equations are called minors of symmetric flattenings or catalecticant minors, and date back at least to Sylvester who coined the term "catalecticant". See [20] for a history.

These equations are usually both too numerous and too few, that is, there are redundancies among them and even all of them usually will not give enough equations to define $\sigma_{r}\left(v_{d}(\mathbb{P} V)\right)$ set-theoretically.

In this paper we

- Describe a large class of new sets of equations for $\sigma_{r}\left(v_{d}(\mathbb{P} V)\right)$, which we call Young Flattenings, that generalize the classical Aronhold invariant, see Proposition 4.1.1.

First author supported by NSF grants DMS-0805782 and 1006353. Second author is member of GNSAGAINDAM.. 
- Show a certain Young Flattening, $Y F_{d, n}$, provides scheme-theoretic equations for a large class of cases where usual flattenings fail, see Theorem 1.2.3.

- Determine cases where flattenings are sufficient to give defining equations (more precisely, scheme-theoretic equations), Theorem 3.2.1. Theorem 3.2.1 is primarily a consequence of work of A. Iarrobino and V. Kanev [25] and Diesel [12].

- Put our results in a larger context by providing a uniform formulation of all known equations for secant varieties via vector bundle methods. We use this perspective to prove some of our results, including a key induction Lemma 6.2.1. The discussion of vector bundle methods is postponed to the latter part of the paper to make the results on symmetric border rank more accessible to readers outside of algebraic geometry.

Here is a chart summarizing what is known about equations of secant varieties of Veronese varieties:

\begin{tabular}{|c|c|c|c|}
\hline case & equations & cuts out & reference \\
\hline$\sigma_{r}\left(v_{2}\left(\mathbb{P}^{n}\right)\right)$ & size $r+1$ minors & ideal & classical \\
\hline$\sigma_{r}\left(v_{d}\left(\mathbb{P}^{1}\right)\right)$ & size $r+1$ minors of any $\phi_{s, d-s}$ & ideal & Gundelfinger, [25] \\
\hline$\sigma_{2}\left(v_{d}\left(\mathbb{P}^{n}\right)\right)$ & $\begin{array}{c}\text { size } 3 \text { minors of any } \\
\phi_{1, d-1} \text { and } \phi_{2, d-2}\end{array}$ & ideal & {$[26]$} \\
\hline$\sigma_{3}\left(v_{3}\left(\mathbb{P}^{n}\right)\right)$ & Aronhold + size 4 minors of $\phi_{1,2}$ & ideal & $\begin{array}{c}\text { Prop. 2.3.1 } \\
\text { Aronhold for } n=2[25]\end{array}$ \\
\hline$\sigma_{3}\left(v_{d}\left(\mathbb{P}^{n}\right)\right), d \geq 4$ & size 4 minors of $\phi_{2,2}$ and $\phi_{1,3}$ & scheme & $\begin{array}{l}\text { Thm.3.2.1 }(1) \\
{[46] \text { for } n=2, d=4}\end{array}$ \\
\hline$\sigma_{4}\left(v_{d}\left(\mathbb{P}^{2}\right)\right)$ & size 5 minors of $\phi_{a, d-a}, a=\left\lfloor\frac{d}{2}\right\rfloor$ & scheme & $\begin{array}{l}\text { Thm. } 3.2 .1(2) \\
{[46] \text { for } d=4}\end{array}$ \\
\hline$\sigma_{5}\left(v_{d}\left(\mathbb{P}^{2}\right)\right), d \geq 6$ and $d=4$ & size 6 minors of $\phi_{a, d-a}, a=\left\lfloor\frac{d}{2}\right\rfloor$ & scheme & $\begin{array}{c}\text { Thm. } 3.2 .1(3) \\
\text { Clebsch for } d=4[25]\end{array}$ \\
\hline$\sigma_{r}\left(v_{5}\left(\mathbb{P}^{2}\right)\right), r \leq 5$ & size $2 r+2$ subPfaffians of $\phi_{31,31}$ & irred.comp. & Thm. 4.2 .7 \\
\hline$\sigma_{6}\left(v_{5}\left(\mathbb{P}^{2}\right)\right)$ & size 14 subPfaffians of $\phi_{31,31}$ & scheme & Thm. 4.2 .7 \\
\hline$\sigma_{6}\left(v_{d}\left(\mathbb{P}^{2}\right)\right), d \geq 6$ & size 7 minors of $\phi_{a, d-a}, a=\left\lfloor\frac{d}{2}\right\rfloor$ & scheme & Thm. 3.2.1 (4) \\
\hline$\sigma_{7}\left(v_{6}\left(\mathbb{P}^{2}\right)\right)$ & symm.flat. + Young flat. & irred.comp. & Thm. 4.2 .9 \\
\hline$\sigma_{8}\left(v_{6}\left(\mathbb{P}^{2}\right)\right)$ & symm.flat. + Young flat. & irred.comp. & Thm. 4.2 .9 \\
\hline$\sigma_{9}\left(v_{6}\left(\mathbb{P}^{2}\right)\right)$ & $\operatorname{det} \phi_{3,3}$ & ideal & classical \\
\hline$\sigma_{j}\left(v_{7}\left(\mathbb{P}^{2}\right)\right), j \leq 10$ & size $2 j+2$ subPfaffians of $\phi_{41,41}$ & irred.comp. & Thm. 1.2 .3 \\
\hline$\sigma_{j}\left(v_{2 \delta}\left(\mathbb{P}^{2}\right)\right), j \leq\left(\begin{array}{c}\delta+1 \\
2\end{array}\right)$ & $\begin{array}{c}\operatorname{rank} \phi_{a, d-a}=\min \left(j,\left(\begin{array}{c}a+2 \\
2\end{array}\right)\right) \\
1 \leq a \leq \delta \\
\text { open and closed conditions }\end{array}$ & scheme & {$[25]$, Thm. $4.1 \mathrm{~A}$} \\
\hline$\sigma_{j}\left(v_{2 \delta+1}\left(\mathbb{P}^{2}\right)\right), j \leq\left(\begin{array}{c}\delta+1 \\
2\end{array}\right)+1$ & $\begin{array}{l}\operatorname{rank} \phi_{a, d-a}=\min \left(j,\left(\begin{array}{c}a+2 \\
2\end{array}\right)\right) \\
1 \leq a \leq \delta \\
\text { open and closed conditions }\end{array}$ & scheme & [25], Thm. 4.5A \\
\hline$\sigma_{j}\left(v_{2 \delta}\left(\mathbb{P}^{n}\right)\right), j \leq\left(\begin{array}{c}\delta+n-1 \\
n\end{array}\right)$ & size $j+1$ minors of $\phi_{\delta, \delta}$ & irred.comp. & [25] Thm. 4.10A \\
\hline$\sigma_{j}\left(v_{2 \delta+1}\left(\mathbb{P}^{n}\right)\right), j \leq\left(\begin{array}{c}\delta+n \\
n\end{array}\right)$ & $\begin{array}{c}\text { size }\left(\begin{array}{c}n \\
a\end{array}\right) j+1 \text { minors of } Y_{d, n}, \\
a=\lfloor n / 2\rfloor \\
\text { if } n=2 a, a \text { odd, }\left(\begin{array}{l}n \\
a\end{array}\right) j+2 \\
\text { subpfaff. of } Y_{d, n}\end{array}$ & irred.comp. & Thm. 1.2 .3 \\
\hline
\end{tabular}

1.2. Young Flattenings. The simplest case of equations for secant varieties is for the space of rank at most $r$ matrices of size $p \times q$, which is the zero set of the minors of size $r+1$. Geometrically let $A=\mathbb{C}^{p}, B=\mathbb{C}^{q}$ and let $\operatorname{Seg}(\mathbb{P} A \times \mathbb{P} B) \subset \mathbb{P}(A \otimes B)$ denote the Segre variety of rank one matrices. Then the ideal of $\sigma_{r}(\operatorname{Seg}(\mathbb{P} A \times \mathbb{P} B))$ is generated by the space of minors of size $r+1$, which is $\wedge^{r+1} A^{*} \otimes \wedge^{r+1} B^{*}$. Now if $X \subset \mathbb{P} W$ is a variety, and there is a linear 
injection $W \rightarrow A \otimes B$ such that $X \subset \sigma_{p}(\operatorname{Seg}(\mathbb{P} A \times \mathbb{P} B))$, then the minors of size $p r+1$ furnish equations for $\sigma_{r}(X)$. Flattenings are a special case of this method where $W=S^{d} V, A=S^{a} V$ and $B=S^{d-a} V$.

When a group $G$ acts linearly on $W$ and $X$ is invariant under the group action, then the equations of $X$ and its secant varieties will be $G$-modules, and one looks for a $G$-module map $W \rightarrow A \otimes B$. Thus one looks for $G$-modules $A, B$ such that $W$ appears in the $G$-module decomposition of $A \otimes B$. We discuss this in detail for $X=v_{d}(\mathbb{P} V)$ in $\S 4$ and in general in $\S 10$ and $\S 11$. For now we focus on a special class of Young flattenings that we describe in elementary language. We begin by reviewing the classical Aronhold invariant.

Example 1.2.1. [The Aronhold invariant] The classical Aronhold invariant is the equation for the hypersurface $\sigma_{3}\left(v_{3}\left(\mathbb{P}^{2}\right)\right) \subset \mathbb{P}^{9}$. Map $S^{3} V \rightarrow\left(V \otimes \wedge^{2} V\right) \otimes\left(V \otimes V^{*}\right)$, by first embedding $S^{3} V \subset V \otimes V \otimes V$, then tensoring with $I d_{V} \in V \otimes V^{*}$, and then skew-symmetrizing. Thus, when $n=2, \phi \in S^{3} V$ gives rise to an element of $\mathbb{C}^{9} \otimes \mathbb{C}^{9}$. In bases, if we write

$$
\begin{aligned}
\phi= & \phi_{000} x_{0}^{3}+\phi_{111} x_{1}^{3}+\phi_{222} x_{2}^{3}+3 \phi_{001} x_{0}^{2} x_{1}+3 \phi_{011} x_{0} x_{1}^{2}+3 \phi_{002} x_{0}^{2} x_{2} \\
& +3 \phi_{022} x_{0} x_{2}^{2}+3 \phi_{112} x_{1}^{2} x_{2}+3 \phi_{122} x_{1} x_{2}^{2}+6 \phi_{012} x_{0} x_{1} x_{2},
\end{aligned}
$$

the corresponding matrix is:

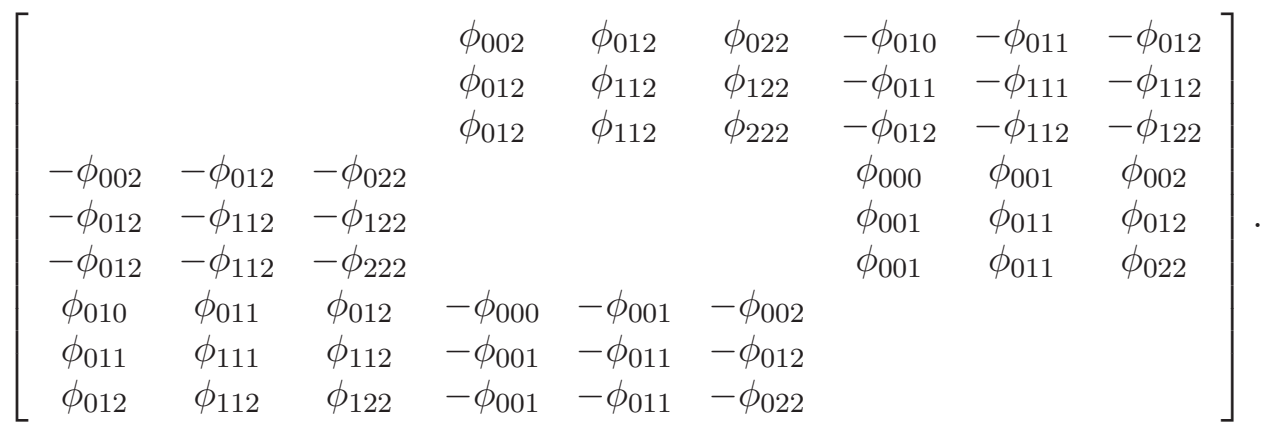

All the principal Pfaffians of size 8 of the this matrix coincide, up to scale, with the classical Aronhold invariant. (Redundancy occurs here is because one should really work with the submodule $S_{21} V \subset V \otimes \wedge^{2} V \simeq V \otimes V^{*}$, where the second identification uses a choice of volume form. The Pfaffian of the map $S_{21} \rightarrow S_{21}$ is the desired equation.)

This construction, slightly different from the one in [40], shows how the Aronhold invariant is analogous to the invariant in $S^{9}\left(\mathbb{C}^{3} \otimes \mathbb{C}^{3} \otimes \mathbb{C}^{3}\right)$ that was discovered by Strassen [48], (see also [39], and the paper [2] by Barth, all in different settings.)

Now consider the inclusion $V \subset \wedge^{k} V^{*} \otimes \wedge^{k+1} V$, given by $v \in V$ maps to the map $\omega \mapsto v \wedge \omega$. In bases one obtains a matrix whose entries are the coefficients of $v$ or zero. In the special case $n+1=2 a+1$ is odd and $k=a$, one obtains a square matrix $K_{n}$, which is skew-symmetric for odd $a$ and symmetric for even $a$. For example, when $n=2$, the matrix is

$$
K_{2}=\left(\begin{array}{ccc}
0 & x_{2} & -x_{1} \\
-x_{2} & 0 & x_{0} \\
x_{1} & -x_{0} & 0
\end{array}\right)
$$

and, when $n=4$, the matrix is 


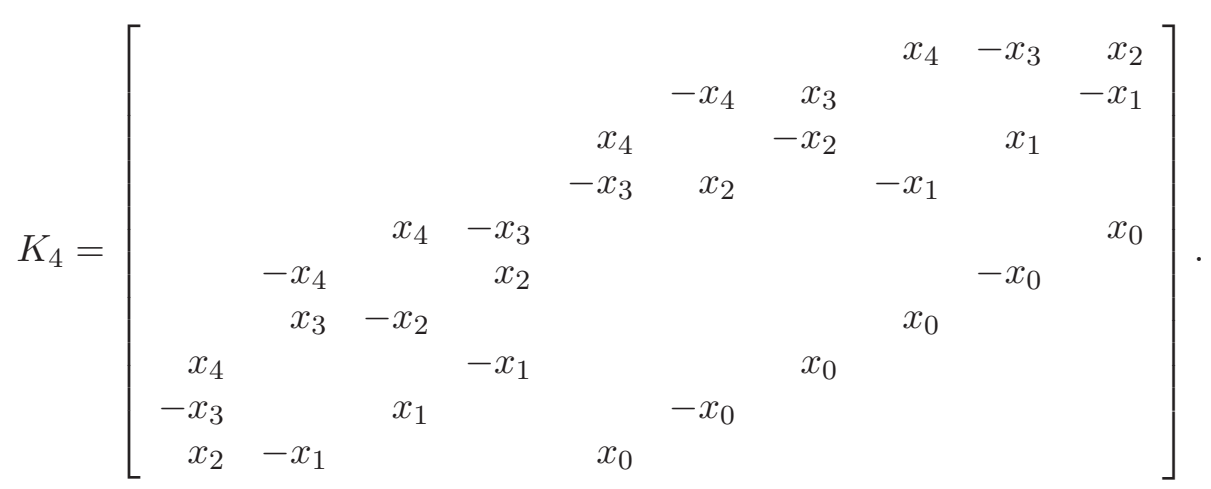

Finally, consider the following generalization of both the Aronhold invariant and the $K_{n}$. Let $a=\left\lfloor\frac{n}{2}\right\rfloor$ and let $d=2 \delta+1$. Map $S^{d} V \rightarrow\left(S^{\delta} V \otimes \wedge^{a} V^{*}\right) \otimes\left(S^{\delta} V \otimes \wedge^{a+1} V\right)$ by first performing the inclusion $S^{d} V \rightarrow S^{\delta} V \otimes S^{\delta} V \otimes V$ and then using the last factor to obtain a map $\wedge^{a} V \rightarrow \wedge^{a+1} V$. We get:

$$
Y F_{d, n}(\phi): S^{\delta} V^{*} \otimes \wedge^{a} V \rightarrow S^{\delta} V \otimes \wedge^{a+1} V
$$

If $n+1$ is odd, the matrix representing $Y F_{d, n}(\phi)$ is skew-symmetic, so we may take Pfaffians instead of minors.

For a decomposable $w^{d} \in S^{d} V$, the map is

$$
\alpha^{\delta} \otimes v_{1} \wedge \cdots \wedge v_{a} \mapsto(\alpha(w))^{\delta} w^{\delta} \otimes w \wedge v_{1} \wedge \cdots \wedge v_{a}
$$

In bases, one obtains a matrix in block form, where the blocks correspond to the entries of $K_{n}$ and the matrices in the blocks are the square catalecticants $\pm\left(\frac{\partial \phi}{\partial x_{i}}\right)_{\delta, \delta}$ in the place of $\pm x_{i}$.

Let

$$
Y F_{d, n}^{r}:=\left\{\phi \in S^{d} V \mid \operatorname{rank}\left(Y F_{d, n}(\phi)\right) \leq\left(\begin{array}{c}
n \\
\left\lfloor\frac{n}{2}\right\rfloor
\end{array}\right) r\right\} .
$$

Two interesting cases are $Y F_{3,2}^{3}=\sigma_{3}\left(v_{3}\left(\mathbb{P}^{2}\right)\right)$ which defines the quartic Aronhold invariant and $Y F_{3,4}^{7}=\sigma_{7}\left(v_{3}\left(\mathbb{P}^{4}\right)\right)$ which defines the invariant of degree 15 considered in [40].

Remark 1.2.2. Just as with the Aronhold invariant above, there will be redundancies among the minors and Pfaffians of $Y F_{d, n}(\phi)$. See $\S 4$ for a description without redundancies.

Theorem 1.2.3. Let $n \geq 2$, let $a=\left\lfloor\frac{n}{2}\right\rfloor$, let $V=\mathbb{C}^{n+1}$, and let $d=2 \delta+1$.

If $r \leq\left(\begin{array}{c}\delta+n \\ n\end{array}\right)$ then $\sigma_{r}\left(v_{d}\left(\mathbb{P}^{n}\right)\right)$ is an irreducible component of $Y F_{d, n}^{r}$, the variety given by the size $\left(\begin{array}{l}n \\ a\end{array}\right) r+1$ minors of $Y F_{d, n}$.

In the case $n=2 a$ with odd $a, Y F_{d, n}$ is skew-symmetric (for any $d$ ) and one may instead take the size $\left(\begin{array}{l}n \\ a\end{array}\right) r+2$ sub-pfaffians of $Y F_{d, n}$.

In the case $n=2 a$ with even $a, Y F_{d, n}$ is symmetric.

The bounds given in Theorem 1.2.3 for $n=2$ are sharp (see Proposition 4.2.3).

1.3. Vector bundle methods. As mentioned above, the main method for finding equations of secant varieties for $X \subset \mathbb{P} V$ is to find a linear embedding $V \subset A \otimes B$, where $A, B$ are vector spaces, such that $X \subset \sigma_{q}(\operatorname{Seg}(\mathbb{P} A \times \mathbb{P} B))$, where $\operatorname{Seg}(\mathbb{P} A \times \mathbb{P} B)$ denotes the Segre variety of rank one elements. What follows is a technique to find such inclusions using vector bundles.

Let $E$ be a vector bundle on $X$ of rank $e$, write $L=\mathcal{O}_{X}(1)$, so $V=H^{0}(X, L)^{*}$. Let $v \in V$ and consider the linear map

$$
A_{v}^{E}: H^{0}(E) \rightarrow H^{0}\left(E^{*} \otimes L\right)^{*}
$$


induced by the natural map

$$
A: H^{0}(E) \otimes H^{0}(L)^{*} \rightarrow H^{0}\left(E^{*} \otimes L\right)^{*}
$$

where $A_{v}^{E}(s)=A(s \otimes v)$. In examples $E$ will be chosen so that both $H^{0}(E)$ and $H^{0}\left(E^{*} \otimes L\right)$ are nonzero, otherwise our construction is vacuous. A key observation (Proposition 5.1.1) is that the size $(r e+1)$ minors of $A_{v}^{E}$ give equations for $\sigma_{r}(X)$.

1.4. Overview. In $\S 2$ we establish notation and collect standard facts that we will need later. In $\S 3$, we first review work of Iarrobino and Kanev [25] and S. Diesel [12], then show how their results imply several new cases where $\sigma_{r}\left(v_{d}(\mathbb{P} V)\right)$ is cut out scheme-theoretically by flattenings (Theorem 3.2.1). In $\S 4$ we discuss Young Flattenings for Veronese varieties. The possible inclusions $S^{d} V \rightarrow S_{\pi} V \otimes S_{\mu} V$ follow easily from the Pieri formula, however which of these are useful is still not understood. In $\S 4.2$ we make a detailed study of the $n=2$ case. The abovementioned Proposition 5.1.1 is proved in $\S 5$, where we also describe simplifications when $(E, L)$ is a symmetric or skew-symmetric pair. We also give a sufficient criterion for $\sigma_{r}(X)$ to be an irreducible component of the equations given by the $(r e+1)$ minors of $A_{v}^{E}$ (Theorem 5.4.3). In $\S 6$ we prove a downward induction lemma (Lemma 6.2.1). We prove Theorem 1.2.3 in $\S 7$, which includes Corollary 7.0.10 on linear systems of hypersurfaces, which may be of interest in its own right. To get explicit models for the maps $A_{v}^{E}$ it is sometimes useful to factor $E$, as described in $\S 8$. In $\S 9$ we explain how to use equations to obtain decompositions of polynomials into sums of powers, illustrating with an algorithm to decompose a general ternary quintic as the sum of seven fifth powers. We conclude, in $\$ 10-11$ with a few brief examples of the construction for homogeneous varieties beyond Veronese varieties.

Acknowledgments. We thank P. Aluffi, who pointed out the refined Bézout theorem 2.4.1. This paper grew out of questions raised at the 2008 AIM workshop Geometry and representation theory of tensors for computer science, statistics and other areas, and the authors thank AIM and the conference participants for inspiration.

\section{BACKGROUND}

2.1. Notation. We work exclusively over the complex numbers. $V, W$ will generally denote (finite dimensional) complex vector spaces. The dual space of $V$ is denoted $V^{*}$. The projective space of lines through the origin of $V$ is denoted by $\mathbb{P} V$. If $A \subset W$ is a subspace $A^{\perp} \subset W^{*}$ is its annihilator, the space of $f \in W^{*}$ such that $f(a)=0 \forall a \in A$.

For a partition $\pi=\left(p_{1}, \ldots, p_{r}\right)$ of $d$, we write $|\pi|=d$ and $\ell(\pi)=r$. If $V$ is a vector space, $S_{\pi} V$ denotes the irreducible $G L(V)$-module determined by $\pi$ (assuming $\operatorname{dim} V \geq \ell(\pi)$ ). In particular $S^{d} W=S_{(d)} W$ and $\wedge^{a} W=S_{1^{a}} W$ are respectively the $d$-th symmetric power and the $a$-th exterior power of $W \cdot S^{d} W=S_{(d)} W$ is also the space of homogeneous polynomials of degree $d$ on $W^{*}$. Given $\phi \in S^{d} W$, Zeros $(\phi) \subset \mathbb{P} W^{*}$ denotes its zero set.

For a subset $Z \subseteq \mathbb{P} W, \hat{Z} \subseteq W \backslash 0$ denotes the affine cone over $Z$. For a projective variety $X \subset$ $\mathbb{P} W, I(X) \subset \operatorname{Sym}\left(W^{*}\right)$ denotes its ideal and $I_{X}$ its ideal sheaf of (regular) functions vanishing at $X$. For a smooth point $z \in X, \hat{T}_{z} X \subset V$ is the affine tangent space and $\hat{N}_{z}^{*} X=\left(\hat{T}_{z} X\right)^{\perp} \subset V^{*}$ the affine conormal space.

We make the standard identification of a vector bundle with the corresponding locally free sheaf. For a sheaf $E$ on $X, H^{i}(E)$ is the $i$-th cohomology space of $E$. In particular $H^{0}(E)$ is the space of global sections of $E$, and $H^{0}\left(I_{Z} \otimes E\right)$ is the space of global sections of $E$ which vanish on a subset $Z \subset X$. According to this notation $H^{0}(\mathbb{P} V, \mathcal{O}(1))=V^{*}$.

If $G / P$ is a rational homogeneous variety and $E \rightarrow G / P$ is an irreducible homogeneous vector bundle, we write $E=E_{\mu}$ where $\mu$ is the highest weight of the irreducible $P$-module inducing $E$. We use the conventions of [4] regarding roots and weights of simple Lie algebras. 
2.2. Flattenings. Given $\phi \in S^{d} V$, write $\phi_{a, d-a} \in S^{a} V \otimes S^{d-a} V$ for the $(a, d-a)$-polarization of $\phi$. We often consider $\phi_{a, d-a}$ as a linear map $\phi_{a, d-a}: S^{a} V^{*} \rightarrow S^{d-a} V$. This notation is compatible with the more general one of Young flattenings that we will introduce in $\S 4$.

If $[\phi] \in v_{d}(\mathbb{P} V)$ then for all $1 \leq a \leq d-1, \operatorname{rank}\left(\phi_{a, d-a}\right)=1$, and the $2 \times 2$ minors of $\phi_{a, d-a}$ generate the ideal of $v_{d}(\mathbb{P} V)$ for any $1 \leq a \leq d-1$. If $[\phi] \in \sigma_{r}\left(v_{d}(\mathbb{P} V)\right)$, then $\operatorname{rank}\left(\phi_{a, d-a}\right) \leq r$, so, the $(r+1) \times(r+1)$ minors of $\phi_{a, d-a}$ furnish equations for $\sigma_{r}\left(v_{d}(\mathbb{P} V)\right)$, i.e.,

$$
\wedge^{r+1}\left(S^{a} V^{*}\right) \otimes \wedge^{r+1}\left(S^{d-a} V^{*}\right) \subset I_{r+1}\left(\sigma_{r}\left(v_{d}(\mathbb{P} V)\right)\right) .
$$

Since $I_{r}\left(\sigma_{r}\left(v_{d}(\mathbb{P} V)\right)\right)=0$, these modules, obtained by symmetric flattenings, also called catalecticant homomorphisms, are among the modules generating the ideal of $\sigma_{r}\left(v_{d}(\mathbb{P} V)\right)$. Geometrically the symmetric flattenings are the equations for the varieties

$$
\operatorname{Rank}_{a, d-a}^{r}\left(S^{d} V\right):=\sigma_{r}\left(\operatorname{Seg}\left(\mathbb{P} S^{a} V \times \mathbb{P} S^{d-a} V\right)\right) \cap \mathbb{P} S^{d} V .
$$

Remark 2.2.1. The equations of $\sigma_{r}\left(v_{2}(\mathbb{P} W)\right) \subset \mathbb{P} S^{2} W$ are those of $\sigma_{r}(S e g(\mathbb{P} W \times \mathbb{P} W))$ restricted to $\mathbb{P} S^{2} W$. Since $S^{2 p} V \subset S^{2}\left(S^{p} V\right)$, when $d=2 a$ we may also describe the symmetric flattenings as the equations for $\sigma_{r}\left(v_{2}\left(\mathbb{P} S^{a} V\right)\right) \cap \mathbb{P} S^{d} V$.

2.3. Inheritance. The purpose of this subsection is to explain why it is only necessary to consider the "primitive" cases of $\sigma_{r}\left(v_{d}\left(\mathbb{P}^{n}\right)\right)$ for $n \leq r-1$.

Let

$$
\begin{aligned}
S u b_{r}\left(S^{d} V\right): & =\mathbb{P}\left\{\phi \in S^{d} V \mid \exists V^{\prime} \subset V, \operatorname{dim} V^{\prime}=r, \phi \in S^{d} V^{\prime}\right\} \\
& =\left\{[\phi] \in \mathbb{P} S^{d} V \mid \operatorname{Zeros}(\phi) \subset \mathbb{P} V^{*} \text { is a cone over a linear space of codimension } r\right\}
\end{aligned}
$$

denote the subspace variety. The ideal of $S u b_{r}\left(S^{d} V\right)$ is generated in degree $r+1$ by all modules $S_{\pi} V^{*} \subset S^{r+1}\left(S^{d} V^{*}\right)$ where $\ell(\pi)>r+1$, see $[49, \S 7.2]$. These modules may be realized explicitly as the $(r+1) \times(r+1)$ minors of $\phi_{1, d-1}$. Note in particular that $\sigma_{r}\left(v_{d}(\mathbb{P} V)\right) \subset S u b_{r}\left(S^{d} V\right)$ and that equality holds for $d \leq 2$ or $r=1$. Hence the equations of $S_{u} b_{r}\left(S^{d} V\right)$ appear among the equations of $\sigma_{r}\left(v_{d}(\mathbb{P} V)\right)$.

Let $X \subset \mathbb{P} W$ be a $G$-variety for some group $G \subset G L(W)$. We recall that a module $M \subset$ $\operatorname{Sym}\left(W^{*}\right)$ defines $X$ set-theoretically if $\operatorname{Zeros}(M)=X$ as a set, that it defines $X$ schemetheoretically if there exists a $\delta$ such that the ideal generated by $M$ equals the ideal of $X$ in all degrees greater than $\delta$, and that $M$ defines $X$ ideal theoretically if the ideal generated by $M$ equals the ideal of $X$.

Proposition 2.3.1 (Symmetric Inheritance). Let $V$ be a vector space of dimension greater than $r$.

Let $M \subset \operatorname{Sym}\left(\left(\mathbb{C}^{r}\right)^{*}\right)$ be a module and let $U=\left[\wedge^{r+1} V^{*} \otimes \wedge^{r+1}\left(S^{d-1} V^{*}\right)\right] \cap S^{r+1}\left(S^{d} V^{*}\right) \subset$ $S^{r+1}\left(S^{d} V^{*}\right)$ be the module generating the ideal of $S u b_{r}\left(S^{d} V\right)$ given by the $(r+1) \times(r+1)$-minors of the flattening $\phi \mapsto \phi_{1, d-1}$.

If $M$ defines $\sigma_{r}\left(v_{d}\left(\mathbb{P}^{r-1}\right)\right)$ set-theoretically, respectively scheme-theoretically, resp. idealtheoretically, let $\tilde{M} \subset \operatorname{Sym}\left(V^{*}\right)$ be the module induced by $M$. Then $\tilde{M}+U$ defines $\sigma_{r}\left(v_{d}(\mathbb{P} V)\right)$ set-theoretically, resp. scheme-theoretically, resp. ideal-theoretically.

See [32, Chapter 8] for a proof.

2.4. Results related to degree. Sometimes it is possible to conclude global information from local equations if one has information about degrees.

We need the following result about excess intersection.

Theorem 2.4.1. Let $Z \subset \mathbb{P}^{n}$ be a variety of codimension $e$ and $L \subset \mathbb{P}^{n}$ a linear subspace of codimension $f$. Assume that $Z \cap L$ has an irreducible component $Y$ of codimension $f+e \leq n$ such that $\operatorname{deg} Y=\operatorname{deg} Z$. Then $Z \cap L=Y$. 
Proof. This is an application of the refined Bézout theorem of [16, Thm. 12.3].

The degrees of $\sigma_{r}\left(v_{d}\left(\mathbb{P}^{2}\right)\right)$ for certain small values of $r$ and $d$ were computed by Ellingsrud and Stromme in [15], see also [25, Rem. 7.20].

Proposition 2.4.2. [15]

(1) $\operatorname{deg}\left(\sigma_{3}\left(v_{4}\left(\mathbb{P}^{2}\right)\right)\right)=112$.

(2) $\operatorname{deg}\left(\sigma_{4}\left(v_{4}\left(\mathbb{P}^{2}\right)\right)=35\right.$.

(3) $\operatorname{deg}\left(\sigma_{6}\left(v_{5}\left(\mathbb{P}^{2}\right)\right)\right)=140$.

(4) $\operatorname{deg}\left(\sigma_{6}\left(v_{6}\left(\mathbb{P}^{2}\right)\right)\right)=28,314$.

Proposition 2.4.3. (see, e.g., [31, Cor. 3.2]) The minimal possible degree of a module in $I\left(\sigma_{r}\left(v_{d}\left(\mathbb{P}^{n}\right)\right)\right)$ is $r+1$.

We recall the following classical formulas, due to C. Segre. For a modern reference see [23].

Proposition 2.4.4.

$$
\begin{gathered}
\operatorname{codim} \sigma_{r}\left(v_{2}\left(\mathbb{P}^{n}\right)\right)=\left(\begin{array}{c}
n-r+2 \\
2
\end{array}\right) \quad \operatorname{deg} \sigma_{r}\left(v_{2}\left(\mathbb{P}^{n}\right)\right)=\prod_{i=0}^{n-r} \frac{\left(\begin{array}{c}
n+1+i \\
n-r-i+1
\end{array}\right)}{\left(\begin{array}{c}
2 i+1 \\
i
\end{array}\right)} \\
\operatorname{codim} \sigma_{r}(G(2, n+1))=\left(\begin{array}{c}
n-2 r+1 \\
2
\end{array}\right) \quad \operatorname{deg} \sigma_{r}(G(2, n+1))=\frac{1}{2^{n-2 r}} \prod_{i=0}^{n-2 r-1} \frac{\left(\begin{array}{c}
n+1+i \\
n-2 r-i
\end{array}\right)}{\left(\begin{array}{c}
2 i+1 \\
i
\end{array}\right)} .
\end{gathered}
$$

2.5. Conormal spaces. The method used to prove a module of equations locally defines $\sigma_{r}\left(v_{d}\left(\mathbb{P}^{n}\right)\right)$ will be to show that the conormal space at a smooth point of the zero set of the module equals the conormal space to $\sigma_{r}\left(v_{d}\left(\mathbb{P}^{n}\right)\right)$ at that point.

Let $A, B$ be vector spaces and let $\operatorname{Seg}(\mathbb{P} A \times \mathbb{P} B) \subset \mathbb{P}(A \otimes B)$ denote the Segre variety, so if $[x] \in \operatorname{Seg}(\mathbb{P} A \times \mathbb{P} B)$, then $x=a \otimes b$ for some $a \in A$ and $b \in B$. One has the affine tangent space $\hat{T}_{[x]} \operatorname{Seg}(\mathbb{P} A \times \mathbb{P} B)=a \otimes B+A \otimes b \subset A \otimes B$, and the affine conormal space $\hat{N}_{[x]}^{*} \operatorname{Seg}(\mathbb{P} A \times$ $\mathbb{P} B)=a^{\perp} \otimes b^{\perp}=\operatorname{ker}(x) \otimes \operatorname{Image}(x)^{\perp} \subset A^{*} \otimes B^{*}$, where in the latter description we think of $a \otimes b: A^{*} \rightarrow B$ as a linear map. Terracini's lemma implies that if $[z] \in \sigma_{r}(\operatorname{Seg}(\mathbb{P} A \times \mathbb{P} B))$ is of rank $r$, then

$$
\hat{N}_{[z]}^{*} \sigma_{r}(\operatorname{Seg}(\mathbb{P} A \times \mathbb{P} B))=\operatorname{ker}(z) \otimes \operatorname{Image}(z)^{\perp} .
$$

In particular, letting $\operatorname{Rank}_{(a, d-a)}^{r}\left(S^{d} V\right) \subset \mathbb{P}\left(S^{d} V\right)$ denote the zero set of the size $(r+1)$-minors of the flattenings $S^{a} V^{*} \rightarrow S^{d-a} V$, one has

Proposition 2.5.1. Let $[\phi] \in \operatorname{Rank}_{(a, d-a)}^{r}\left(S^{d} V\right)$ be a sufficiently general point, then

$$
\hat{N}_{[\phi]}^{*} \operatorname{Rank}_{(a, d-a)}^{r}\left(S^{d} V\right)=\operatorname{ker}\left(\phi_{a, d-a}\right) \circ \operatorname{Image}\left(\phi_{a, d-a}\right)^{\perp} \subset S^{d} V^{*} .
$$

Now let $\left[y^{d}\right] \in v_{d}(\mathbb{P} V)$, then

$$
\begin{aligned}
\hat{N}_{\left[y^{d}\right]}^{*} v_{d}(\mathbb{P} V) & =\left\{P \in S^{d} V^{*} \mid P(y)=0, d P_{y}=0\right\} \\
& =S^{d-2} V^{*} \circ S^{2} y^{\perp} \\
& =\left\{P \in S^{d} V^{*} \mid \operatorname{Zeros}(P) \text { is singular at }[y]\right\} .
\end{aligned}
$$

Applying Terracini's lemma yields:

Proposition 2.5.2. Let $[\phi]=\left[y_{1}^{d}+\cdots+y_{r}^{d}\right] \in \sigma_{r}\left(v_{d}(\mathbb{P} V)\right)$. Then

$$
\begin{aligned}
\hat{N}_{[\phi]}^{*} \sigma_{r}\left(v_{d}(\mathbb{P} V)\right) & \subseteq\left(S^{d-2} V^{*} \circ S^{2} y_{1}{ }^{\perp}\right) \cap \cdots \cap\left(S^{d-2} V^{*} \circ S^{2} y_{r}{ }^{\perp}\right) \\
& =\left\{P \in S^{d} V^{*} \mid \operatorname{Zeros}(P) \text { is singular at }\left[y_{1}\right], \ldots,\left[y_{r}\right]\right\}
\end{aligned}
$$


and equality holds if $\phi$ is sufficiently general.

\section{Symmetric Flattenings (Catalecticant minors)}

3.1. Review of known results. The following result dates back to the work of Sylvester and Gundelfinger.

Theorem 3.1.1. (see, e.g., [25, Thm. 1.56]) The ideal of $\sigma_{r}\left(v_{d}\left(\mathbb{P}^{1}\right)\right)$ is generated in degree $r+1$ by the size $r+1$ minors of $\phi_{u, d-u}$ for any $r \leq u \leq d-r$, i.e., by any of the modules $\wedge^{r+1} S^{u} \mathbb{C}^{2} \otimes \wedge^{r+1} S^{d-u} \mathbb{C}^{2}$. The variety $\sigma_{r}\left(v_{d}\left(\mathbb{P}^{1}\right)\right)$ is projectively normal and arithmetically Cohen-Macaulay, its singular locus is $\sigma_{r-1}\left(v_{d}\left(\mathbb{P}^{1}\right)\right)$, and its degree is $\left(\begin{array}{c}d-r+1 \\ r\end{array}\right)$.

Corollary 3.1.2 (Kanev, [26]). The ideal of $\sigma_{2}\left(v_{d}\left(\mathbb{P}^{n}\right)\right)$ is generated in degree 3 by the 3 by 3 minors of the $(1, d-1)$ and $(2, d-2)$ flattenings.

Proof. Apply Proposition 2.3.1 to Theorem 3.1.1 in the case $r=2$.

Remark 3.1.3. C. Raicu [43] recently proved that in Corollary 3.1.2 it is possible to replace the $(2, d-2)$ flattening with any $(i, d-i)$-flattening such that $2 \leq i \leq d-2$.

Theorem 3.1.4. (see $\left[25\right.$, Thms. 4.5A, 4.10A]) Let $n \geq 3$, and let $V=\mathbb{C}^{n+1}$. Let $\delta=\left\llcorner\frac{d}{2}\right\lrcorner$, $\delta^{\prime}=\left\ulcorner\frac{d}{2}\right\urcorner$. If $r \leq\left(\begin{array}{c}\delta-1+n \\ n\end{array}\right)$ then $\sigma_{r}\left(v_{d}(\mathbb{P} V)\right)$ is an irreducible component of $\sigma_{r}\left(\operatorname{Seg}\left(\mathbb{P} S^{\delta} V \times\right.\right.$ $\left.\left.\mathbb{P} S^{\delta^{\prime}} V\right)\right) \cap \mathbb{P}\left(S^{d} V\right)$. In other words, $\sigma_{r}\left(v_{d}\left(\mathbb{P}^{n}\right)\right)$ is an irreducible component of the size $(r+1)$ minors of the $\left(\delta, \delta^{\prime}\right)$-flattening.

The bound obtained in this theorem is the best we know of for even degree, apart from some cases of small degree listed below. Theorem 1.2.3 is an improvement of this bound in the case of odd degree.

When $\operatorname{dim} V=3$ then a Zariski open subset of $\sigma_{r}\left(v_{d}(\mathbb{P} V)\right)$ can be characterized by open and closed conditions, that is, with the same assumptions of Theorem 3.1.4: If the rank of all $(a, d-a)$-flattenings computed at $\phi$ is equal to $\min \left(r,\left(\begin{array}{c}a+2 \\ 2\end{array}\right)\right)$ then $\phi \in \sigma_{r}\left(v_{d}(\mathbb{P} V)\right)$ and the general element of $\sigma_{r}\left(v_{d}(\mathbb{P} V)\right)$ can be described in this way (see [25, Thm. 4.1A]).

Remark 3.1.5. The statement of Theorem 3.1.4 cannot be improved, in the sense that that the zero locus of the flattenings usually is reducible. Consider the case $n=2, d=8, r=10$, in this case the $10 \times 10$ flattenings of $\phi_{4,4}$ define a variety with at least two irreducible components, one of them is $\sigma_{10}\left(v_{8}\left(\mathbb{P}^{2}\right)\right)$ (see [25, Ex. 7.11]).

Proposition 3.1.6. Assume $\sigma_{r}\left(v_{d}\left(\mathbb{P}^{n}\right)\right)$ is not defective and $r<\frac{1}{n+1}\left(\begin{array}{c}n+d \\ d\end{array}\right)$ so that $\sigma_{r}\left(v_{d}\left(\mathbb{P}^{n}\right)\right)$ is not the ambient space. Let $\delta=\left\llcorner\frac{d}{2}\right\lrcorner, \delta^{\prime}=\left\ulcorner\frac{d}{2}\right\urcorner$. There are non-trivial equations from flattenings iff $r<\left(\begin{array}{c}\delta+n \\ \delta\end{array}\right)$. (The defective cases where $r$ is allowed to be larger are understood as well.)

Proof. The right hand side is the size of the maximal minors of $\phi_{\delta, \delta^{\prime}}$, which give equations for $\sigma_{\left(\begin{array}{c}\delta+n \\ \delta\end{array}\right)-1}\left(v_{d}\left(\mathbb{P}^{n}\right)\right)$.

For example, when $n=3$ and $d=2 \delta, \sigma_{r}\left(v_{d}\left(\mathbb{P}^{3}\right)\right)$ is an irreducible component of the zero set of the flattenings for $r \leq\left(\begin{array}{c}\delta+2 \\ 3\end{array}\right)$, the flattenings give some equations up to $r \leq\left(\begin{array}{c}\delta+3 \\ 3\end{array}\right)$, and there are non-trivial equations up to $r \leq \frac{1}{4}\left(\begin{array}{c}2 \delta+3 \\ 3\end{array}\right)$.

We will have need of more than one symmetric flattening, so we make the following definitions, following [25], but modifying the notation: 
Definition 3.1.7. Fix a sequence $\vec{r}:=\left(r_{1}, \ldots, r_{\left\llcorner\frac{d}{2}\right\lrcorner}\right)$ and let

$$
\begin{aligned}
\operatorname{SFlat}_{\vec{r}}\left(S^{d} W\right):= & \left\{\phi \in S^{d} W \mid \operatorname{rank}\left(\phi_{j, d-j}\right) \leq r_{j}, j=1, \ldots,\left\llcorner\frac{d}{2}\right\lrcorner\right\} \\
& =\left[\bigcap_{j=1}^{\left\llcorner\frac{d}{2}\right\lrcorner} \sigma_{r_{j}}\left(\operatorname{Seg}\left(\mathbb{P} S^{j} W \times \mathbb{P} S^{d-j} W\right)\right)\right] \cap S^{d} W
\end{aligned}
$$

Call a sequence $\vec{r}$ admissible if there exists $\phi \in S^{d} W$ such that $\operatorname{rank}\left(\phi_{j, d-j}\right)=r_{j}$ for all $j=1, \ldots,\left\llcorner\frac{d}{2}\right\lrcorner$. It is sufficient to consider $\vec{r}$ that are admissible because if $\vec{r}$ is non-admissible, the zero set of $S F l a t_{\vec{r}}$ will be contained in the union of admissible $S F l a t$ 's associated to smaller $\vec{r}$ s in the natural partial order. Note that $\operatorname{SFlat}_{\vec{r}}\left(S^{d} W\right) \subseteq S u b_{r_{1}}\left(S^{d} W\right)$.

Even if $\vec{r}$ is admissible, it still can be the case that $S F l a t_{\vec{r}}\left(S^{d} W\right)$ is reducible. For example, when $\operatorname{dim} W=3$ and $d \geq 6$, the zero set of the size 5 minors of the $(2, d-2)$-flattening has two irreducible components, one of them is $\sigma_{4}\left(v_{d}\left(\mathbb{P}^{2}\right)\right)$ and the other has dimension $d+6[25$, Ex. $3.6]$.

To remedy this, let $\vec{r}$ be admissible, and consider

$$
\operatorname{SFlat}_{\vec{r}}^{0}\left(S^{d} W\right):=\left\{\phi \in S^{d} W \mid \operatorname{rank}\left(\phi_{j, d-j}\right)=r_{j}, j=1, \ldots,\left\llcorner\frac{d}{2}\right\lrcorner\right\}
$$

and let

$$
\operatorname{Gor}(\vec{r}):=\overline{S F l a t} \frac{\vec{r}\left(S^{d} W\right)}{}
$$

Remark 3.1.8. In the commutative algebra literature (e.g. [12, 25]), "Gor" is short for Gorenstein, see [25, Def. 1.11] for a history.

Unfortunately, defining equations for $\operatorname{Gor}(\vec{r})$ are not known. One can test for membership of $S F l a t_{\vec{r}}^{0}\left(S^{d} W\right)$ by checking the required vanishing and non-vanishing of minors.

Theorem 3.1.9. [12, Thm 1.1] If $\operatorname{dim} W=3$, and $\vec{r}$ is admissible, then Gor $(\vec{r})$ is irreducible.

Theorem 3.1.9 combined with Theorem 3.1.4 allows one to extend the set of secant varieties of Veronese varieties defined by flattenings.

\subsection{Consequences of Theorems 3.1.9 and 3.1.4.}

Theorem 3.2.1. The following varieties are defined scheme-theoretically by minors of flattenings:

(1) Let $d \geq 4$. The variety $\sigma_{3}\left(v_{d}\left(\mathbb{P}^{n}\right)\right)$ is defined scheme-theoretically by the $4 \times 4$ minors of the $(1, d-1)$ and $\left(\left\lfloor\frac{d}{2}\right\rfloor, d-\left\lfloor\frac{d}{2}\right\rfloor\right)$ flattenings.

(2) For $d \geq 4$ the variety $\sigma_{4}\left(v_{d}\left(\mathbb{P}^{2}\right)\right)$ is defined scheme-theoretically by the $5 \times 5$ minors of the $\left(\left\lfloor\frac{\bar{d}}{2}\right\rfloor, d-\left\lfloor\frac{d}{2}\right\rfloor\right)$ flattenings.

(3) For $d \geq 6$ the variety $\sigma_{5}\left(v_{d}\left(\mathbb{P}^{2}\right)\right)$ is defined scheme-theoretically by the $6 \times 6$ minors of the $\left(\left\lfloor\frac{d}{2}\right\rfloor, d-\left\lfloor\frac{d}{2}\right\rfloor\right)$ flattenings.

(4) Let $d \geq 6$. The variety $\sigma_{6}\left(v_{d}\left(\mathbb{P}^{2}\right)\right)$ is defined scheme-theoretically by the $7 \times 7$ minors of the $\left(\left\lfloor\frac{d}{2}\right\rfloor, d-\left\lfloor\frac{d}{2}\right\rfloor\right)$ flattenings.

Remark 3.2.2. In the recent preprint [6] it is proved that the variety $\sigma_{r}\left(v_{d}\left(\mathbb{P}^{n}\right)\right)$ is defined settheoretically by the $(r+1) \times(r+1)$ minors of the $(i, d-i)$ flattenings for $n \leq 3,2 r \leq d$ and $r \leq i \leq d-r$. Schreyer proved (2) in the case $d=4$, [46, Thm. 2.3].

By [47, Thm 4.2], when $n \leq 2, \operatorname{rank}\left(\phi_{s, d-s}\right)$ is nondecreasing in $s$ for $1 \leq s \leq\left\lfloor\frac{d}{2}\right\rfloor$. We will use this fact often in this section. 
To prove each case, we simply show the scheme defined by the flattenings in the hypotheses coincides with the scheme of some $\operatorname{Gor}(\vec{r})$ with $\vec{r}$ admissible and then Theorem 3.1.4 combined with Theorem 3.1.9 implies the result. The first step is to determine which $\vec{r}$ are admissible.

Lemma 3.2.3. The only admissible sequences $\vec{r}$ with $r_{1}=3$ and $r_{i} \leq 5$ are

(1) $(3, \ldots, 3)$ for $d \geq 3$,

(2) $(3,4,4, \ldots, 4)$ for $d \geq 4$,

(3) $(3,5,5, \ldots, 5)$ for $d \geq 4$,

(4) $(3,4,5, \ldots, 5)$ for $d \geq 6$.

Lemma 3.2.3 is proved below. We first show how it implies the results above, by showing in each case a general $\phi$ satisfying the hypotheses of the theorem must be in the appropriate SFlat $t_{\vec{r}}^{0}$ with $\vec{r}$ admissible. Since $\sigma_{r}\left(v_{d}(\mathbb{P} V)\right)$ is irreducible, one concludes.

Proof of (1). We may assume that $\operatorname{rank}\left(\phi_{1, d-1}\right)=3$, otherwise we are in the case of two variables. By inheritance, it is sufficient to prove the result for $n=2$. In this case, if $\operatorname{rank}\left(\phi_{a, d-a}\right) \leq 3$ for $a=\left\lfloor\frac{d}{2}\right\rfloor$, then $\operatorname{rank}\left(\phi_{a, d-a}\right) \leq 3$ for all $a$ such that $2 \leq a \leq d-2$, and $\vec{r}=(3, \ldots, 3)$ is admissible .

Proof of (2). We may assume that $\operatorname{rank} \phi_{1, d-1}=3$. Let $d=4$. If $\operatorname{rank}\left(\phi_{2, d-2}\right) \leq 4$ then by Lemma 3.2.3 the only possible $\vec{r}$ are $(3,3)$ and $(3,4)$. The first case corresponds to $\sigma_{3}\left(v_{4}\left(\mathbb{P}^{2}\right)\right) \subset$ $\sigma_{4}\left(v_{4}\left(\mathbb{P}^{2}\right)\right)$ and the second case is as desired. The case $d \geq 5$ is analogous.

Proof of (3). If $\phi \notin \sigma_{4}\left(v_{d}(\mathbb{P} W)\right)$, then $\phi \in \operatorname{Gor}(3,5, \ldots, 5)=\sigma_{5}\left(v_{d}\left(\mathbb{P}^{2}\right)\right)$ or $\phi \in \operatorname{Gor}(3,4,5, \ldots, 5)$.

It remains to show $\operatorname{Gor}(3,4,5, \ldots, 5) \subset \sigma_{5}\left(v_{d}\left(\mathbb{P}^{2}\right)\right)$. To prove this we generalize the argument of $[25$, Ex. 5.72].

Let $\phi \in S F l a t_{3,4,5, \ldots, 5}^{0}\left(S^{d} W\right)$ and consider the kernel of $\phi_{2, d-2}$ as a 2-dimensional space of plane conics. If the base locus is a zero-dimensional scheme of length four, then $\phi \in \sigma_{4}\left(v_{d}\left(\mathbb{P}^{2}\right)\right)$, and $\operatorname{rank}\left(\phi_{3, d-3}\right) \leq 4$ which contradicts $\phi \in$ SFlat $_{3,4,5, \ldots, 5}^{0}\left(S^{d} W\right)$. Thus the base locus has dimension one and, in convenient coordinates, the kernel of $\phi_{2, d-2}$ is $\langle x y, x z\rangle$. It follows that $\phi=x^{d}+\psi(y, z)$ and from $\operatorname{rank}\left(\phi_{a, d-a}\right)=5$ it follows $\operatorname{rank}\left(\psi_{a, d-a}\right)=4$. Since $\psi$ has two variables, this implies $\psi \in \sigma_{4}\left(v_{d}\left(\mathbb{P}^{2}\right)\right)$ and $\phi \in \sigma_{5}\left(v_{d}\left(\mathbb{P}^{2}\right)\right)$ as required.

Proof of (4). If the sequence of ranks of $\phi_{a, d-a}$ is $\vec{r}=(1,3,6, \ldots, 6,3,1)$ then $\phi \in \operatorname{Gor}(\vec{r})=$ $\sigma_{6}\left(v_{d}\left(\mathbb{P}^{n}\right)\right)$.

Otherwise $\operatorname{rank}\left(\phi_{2, d-2}\right) \leq 5$. In this case, by an extension of Lemma 3.2.3, there are just two other possibilities for $\vec{r}$, namely $\vec{r}_{1}=(1,3,5,6 \ldots, 6,5,3,1)$ and $\vec{r}_{2}=(1,3,4,5,6 \ldots, 6,5,4,3,1)$ for $d \geq 8$.

Consider $\phi \in \vec{r}_{2}$. As above, we may assume that $\operatorname{ker}\left(\phi_{2, d-2}\right)=<x y, x z>$. It follows that $\phi=x^{d}+\psi(y, z)$. If if $d \leq 9$ then $\psi \in \sigma_{5}\left(v_{d}\left(\mathbb{P}^{2}\right)\right)$. If $d \geq 10, \operatorname{rank}\left(\psi_{5, d-5}\right)=5$ because $\operatorname{rank}\left(\phi_{5, d-5}\right)=6$. Since $\psi$ has two variables, $\psi \in \sigma_{5}\left(v_{d}\left(\mathbb{P}^{2}\right)\right)$. Thus $\phi \in \sigma_{6}\left(v_{d}\left(\mathbb{P}^{2}\right)\right)$ as desired.

Consider $\phi \in \vec{r}_{1}$. The cases $d=6$ and $d \geq 8$ respectively follow from [25, Ex. 5.72] and [25, Thm. 5.71 (i)]. Here is a uniform argument for all $d \geq 6$ : There is a conic $C$ in the kernel of $\phi_{2, d-2}$. The four dimensional space of cubics which is the kernel of $\phi_{3, d-3}$ is generated by $C \cdot L$ where $L$ is any line and a cubic $F$. Then the 6 points of $C \cap F$ are the base locus, by [25, Cor 5.69], so $\phi \in \sigma_{6}\left(v_{d}\left(\mathbb{P}^{2}\right)\right)$.

Let $\operatorname{dim} W=3$. We will need the following facts to prove Lemma 3.2.3:

(1) For $\phi \in S^{d} W$, consider the ideal $\phi^{\perp}$ generated in degrees $\leq d$, by $\operatorname{ker}\left(\phi_{a, d-a}\right) \subset S^{a} W^{*}$, $1 \leq a \leq d$, and the ring $A_{\phi}:=\operatorname{Sym}\left(W^{*}\right) / \phi^{\perp}$. Note that the values of the Hilbert function of $A_{\phi}, H_{A_{\phi}}(j)$, are $T(\vec{r}):=\left(1, r_{1}, \ldots, r_{\left\llcorner\frac{d}{2}\right\lrcorner}, r_{\left\llcorner\frac{d}{2}\right\lrcorner}, \ldots, r_{1}, 1,0, \ldots, 0\right)$, where recall that $r_{j}=\operatorname{rank}\left(\phi_{j, d-j}\right)$. 
(2) [5, Thm. 2.1] The ring $A_{\phi}$ has a minimal free resolution of the form

$$
\begin{array}{r}
0 \longrightarrow \operatorname{Sym}\left(W^{*}\right)(-d-3) \stackrel{g^{*}}{\longrightarrow} \oplus_{i=1}^{u} \operatorname{Sym}\left(W^{*}\right)\left(-d+3-q_{i}\right) \\
\stackrel{h}{\longrightarrow} \oplus_{i=1}^{u} \operatorname{Sym}\left(W^{*}\right)\left(-q_{i}\right) \stackrel{g}{\longrightarrow} \operatorname{Sym}\left(W^{*}\right)(0) \rightarrow A_{\phi} \rightarrow 0
\end{array}
$$

where the $q_{j}$ are non-decreasing. Here $\operatorname{Sym}\left(W^{*}\right)(j)$ denotes $\operatorname{Sym}\left(W^{*}\right)$ with the labeling of degrees shifted by $j$, so $\operatorname{dim}\left(\operatorname{Sym}\left(W^{*}\right)(j)\right)_{d}=\left(\begin{array}{c}d+j+2 \\ 2\end{array}\right)$.

(3) Moreover [12, Thm 1.1] there is a unique resolution with the properties $q_{1} \leq \frac{d+3}{2}$, $q_{i}+q_{u-i+2}=d+2,2 \leq i \leq \frac{u+1}{2}$ having $T(\vec{r})$ as the values of its Hilbert function.

(4) Recall that $H_{A_{\phi}}(j)$ is also the alternating sum of the dimensions of the degree $j$ term in (5) (forgetting the last term). Thus the $q_{j}$ determine the $r_{i}$.

(5) $\left[12\right.$, Thm. 3.3] Letting $j_{0}$ be the smallest $j$ such that $\phi_{j, d-j}$ is not injective, then $u=$ $2 j_{0}+1$ in the resolution above.

Remark 3.2.4. Although we do not need these facts from [5] here, we note that above: $h$ is skewsymmetric, $g$ is defined by the principal sub-Pfaffians of $h, A_{\phi}$ is an Artinian Gorenstein graded $\mathbb{C}$-algebra and any Artinian Gorenstein graded $\mathbb{C}$-algebra is isomorphic to $A_{\phi}$ for a polynomial $\phi$ uniquely determined up to constants. The ring $A_{\phi}$ is called the apolar ring of $\phi$, the resolution above is called saturated.

Proof of Lemma 3.2.3. Let $\vec{r}$ be a sequence as in the assumptions. Since $\operatorname{dim} S^{2} \mathbb{C}^{3}=6>5$, $u=5$ by Fact 3.2(5). Consider the unique resolution satisfying Fact 3.2(3) having $T(\vec{r})$ as Hilbert function. The number of generators in degree 2 is the number of the $q_{i}$ equal to 2 . We must have $q_{1}=2$, otherwise, by computing the Hilbert function, $H\left(A_{\phi}\right)(2)=6$ contrary to assumption. The maximal number of generators in degree 2 is three, otherwise we would have $2+2=q_{4}+q_{5-4+2}=d+2$, a contradiction.

If there are three generators in degree 2 then $q_{i}=(2,2,2, d, d)$, and computing the Hilbert function via the Euler characteristic, we are in case (1) of the Lemma.

If there are two generators in degree 2 then there are the two possibilities $q_{i}=(2,2,3, n-1, n)$ $(\operatorname{case}(2))$ or $q_{i}=(2,2,4, n-2, n)$ (case (4)). Note that $q_{i}=(2,2,5, n-3, n)$ for $n \geq 8$ is impossible because otherwise $\vec{r}=(1,3,4,5,6, \ldots)$ contrary to assumption. By similar arguments one shows that there are no other possibilities.

If there is one generator in degree 2 then $q_{i}=(2,3,3, n-1, n-1)($ case $(3))$.

Proposition 3.2.5. Let $\operatorname{dim} V=3$ and $p \geq 2$. If the variety $\sigma_{r}\left(v_{2 p}(\mathbb{P} V)\right)$ is an irreducible component of $\sigma_{r}\left(v_{2}\left(\mathbb{P} S^{p} V\right)\right) \cap \mathbb{P} S^{2 p} V$ then $r \leq \frac{1}{2} p(p+1)$ or $(p, r)=(2,4)$ or $(3,9)$.

Proof. Recall that for $(p, r) \neq(2,5)$

$$
\operatorname{codim} \sigma_{r}\left(v_{2 p}(\mathbb{P} V)\right)=\left(\begin{array}{c}
2 p+2 \\
2
\end{array}\right)-3 r
$$

and from Proposition 2.4.4 every irreducible component $X$ of $\sigma_{r}\left(v_{2}\left(\mathbb{P} S^{p} V\right)\right) \cap \mathbb{P} S^{2 p} V$ satisfies

$$
\operatorname{codim} X \leq \frac{1}{2}\left[\left(\begin{array}{c}
p+2 \\
2
\end{array}\right)-r\right]\left[\left(\begin{array}{c}
p+2 \\
2
\end{array}\right)-r+1\right]
$$

The result follows by solving the inequality.

For the case $(p, r)=(2,4)$ see Theorem 3.2.1(2) and for the case $(p, r)=(3,9)$ see Theorem 4.2.9.

Corollary 3.2.6. $\operatorname{deg} \sigma_{\left(\begin{array}{c}p+1 \\ 2\end{array}\right)}\left(v_{2 p}\left(\mathbb{P}^{2}\right)\right) \leq \prod_{i=0}^{p} \frac{\left(\begin{array}{c}(p+2)(p+1) / 2+i \\ p-i+1\end{array}\right)}{\left(\begin{array}{c}2 i+1 \\ i\end{array}\right)}$. 
If equality holds then $\sigma_{\left(\begin{array}{c}p+1 \\ 2\end{array}\right)}\left(v_{2 p}\left(\mathbb{P}^{2}\right)\right)$ is given scheme-theoretically by the $\left(\frac{1}{2}\right)\left(p^{2}+p+2\right)$ minors of the $(p, p)$-flattening.

The values of the right hand side of the inequality for $p=1, \ldots 4$ are respectively 4,112 , 28314,81662152 .

Proof. The right hand side is $\operatorname{deg} \sigma_{\left(\begin{array}{c}p+1 \\ 2\end{array}\right)}\left(v_{2}\left(\mathbb{P}^{p(p+3) / 2}\right)\right)$ by Proposition 2.4.4. If $\phi \in \sigma_{\left(\begin{array}{c}p+1 \\ 2\end{array}\right)}\left(v_{2 p}\left(\mathbb{P}^{2}\right)\right)$ then $\operatorname{rank}\left(\phi_{p, p}\right) \leq\left(\begin{array}{c}p+1 \\ 2\end{array}\right)$. This means $\sigma_{\left(\begin{array}{c}p+1 \\ 2\end{array}\right)}\left(v_{2 p}\left(\mathbb{P}^{2}\right)\right)$ is contained in a linear section of $\sigma_{\left(\begin{array}{c}p+1 \\ 2\end{array}\right)}\left(v_{2}\left(\mathbb{P}^{p(p+3) / 2}\right)\right)$, and by Theorem 3.1.4 it is a irreducible component of this linear section. The result follows by the refined Bezout Theorem 2.4.1.

Equality holds in Corollary 3.2.6 for the cases $p=1,2,3$ by Proposition 2.4.2. The case $p=1$ corresponds to the quadratic Veronese surface and the cases $p=2,3$ will be considered respectively in Thm.3.2.1 (1) and Theorem 4.2.8. For $p \geq 4$ these numbers are out of the range of the results in [15] (the points are too few to be fixed points of a torus action) and we do not know if equality holds.

\section{Young Flattenings for Veronese VARIETiES}

4.1. Preliminaries. In what follows we fix $n+1=\operatorname{dim} V$ and endow $V$ with a volume form and thus identify (as $S L(V)$-modules) $S_{\left(p_{1}, \ldots, p_{n+1}\right)} V$ with $S_{\left(p_{1}-p_{n+1}, p_{2}-p_{n+1}, \ldots, p_{n}-p_{n+1}, 0\right)} V$. We will say $\left(p_{1}-p_{n}, p_{2}-p_{n}, \ldots, p_{n-1}-p_{n}, 0\right)$ is the reduced partition associated to $\left(p_{1}, \ldots, p_{n}\right)$.

The Pieri formula states that $S_{\pi} V^{*} \subset S_{\nu} V^{*} \otimes S^{d} V^{*}$ iff the Young diagram of $\pi$ is obtained by adding $d$ boxes to the Young diagram of $\nu$, with no two boxes added to the same column. Moreover, if this occurs, the multiplicity of $S_{\pi} V^{*}$ in $S_{\nu} V^{*} \otimes S^{d} V^{*}$ is one.

We record the basic observation that the dual $S L(V)$-module to $S_{\pi} V$ is obtained by considering the complement to $\pi$ in the $\ell(\pi) \times \operatorname{dim} V$ rectangle and rotating it to give a Young diagram whose associated partition we denote $\pi^{*}$.

Say $S_{\pi} V^{*} \subset S_{\nu} V \otimes S^{d} V^{*}$ and consider the map $S^{d} V \rightarrow S_{\pi} V \otimes S_{\nu} V^{*}$. Let $S_{\mu} V=S_{\nu} V^{*}$ where $\mu$ is the reduced partition with this property. We obtain an inclusion $S^{d} V \rightarrow S_{\pi} V \otimes S_{\mu} V$.

Given $\phi \in S^{d} V$, let $\phi_{\pi, \mu} \in S_{\pi} V \otimes S_{\mu} V$ denote the corresponding element. If $S_{\mu} V=S_{\nu} V^{*}$ as an $S L(V)$-module, we will also write $\phi_{\pi, \nu^{*}}=\phi_{\pi, \mu}$ when we consider it as a linear map $S_{\nu} V \rightarrow S_{\pi} V$.

The following proposition is an immediate consequence of the subadditivity for ranks of linear maps.

Proposition 4.1.1. Rank conditions on $\phi_{\pi, \mu}$ provide equations for the secant varieties of $v_{d}(\mathbb{P} V)$ as follows: Let $\left[x^{d}\right] \in v_{d}(\mathbb{P} V)$. Say $\operatorname{rank}\left(x_{\pi, \mu}^{d}\right)=t$. If $[\phi] \in \sigma_{r}\left(v_{d}(\mathbb{P} V)\right)$, then $\operatorname{rank}\left(\phi_{\pi, \mu}\right) \leq r t$. Thus if $r+1 \leq \min \left\{\operatorname{dim} S_{\pi} V, \operatorname{dim} S_{\mu} V\right\}$, the $(r t+1) \times(r t+1)$ minors of $\phi_{\pi, \mu}$ provide equations for $\sigma_{r}\left(v_{d}(\mathbb{P} V)\right)$, i.e.,

$$
\wedge^{r t+1}\left(S_{\pi} V^{*}\right) \otimes \wedge^{r t+1}\left(S_{\nu} V^{*}\right) \subset I_{r t+1}\left(\sigma_{r}\left(v_{d}(\mathbb{P} V)\right)\right) .
$$

Remark 4.1.2. From the inclusion $S^{d} V \subset V \otimes S^{a} V \otimes S^{d-a-1} V$ we obtain $v_{d}(\mathbb{P} V) \subset S e g(\mathbb{P} V \times$ $\left.\mathbb{P} S^{a} V \times \mathbb{P} S^{d-a-1} V\right)$. Strassen's equations for $\sigma_{n+s}\left(\operatorname{Seg}\left(\mathbb{P}^{2} \times \mathbb{P}^{n-1} \times \mathbb{P}^{n-1}\right)\right)$ give equations for secant varieties of Veronese varieties via this three-way flattening. The Aronhold equation comes from the Strassen equations for $\sigma_{3}\left(\operatorname{Seg}\left(\mathbb{P}^{2} \times \mathbb{P}^{2} \times \mathbb{P}^{2}\right)\right)$.

So far we have not obtained any new equations using three way symmetric flattenings and Young flattenings. We mention three-way symmetric flattenings because they may be useful in future investigations, especially when further modules of equations for secant varieties of triple Segre products are found. 
Let

$$
Y \operatorname{Flat}_{\pi, \mu}^{t}\left(S^{d} V\right):=\overline{\left\{\phi \in S^{d} V \mid \operatorname{rank}\left(\phi_{\pi, \mu}\right) \leq t\right\}}=\sigma_{t}\left(\operatorname{Seg}\left(\mathbb{P} S_{\pi} V \times \mathbb{P} S_{\mu} V\right)\right) \cap \mathbb{P} S^{d} V .
$$

Remark 4.1.3. Recall $Y F_{d, n}^{r}$ from $\S 1.2$ whose description had redundancies. We can now give an irredundant description of its defining equations:

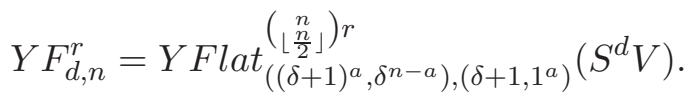

This is a consequence of Schur's Lemma, because the module $S_{\left(\delta+1,1^{a}\right)} V$ is the only one appearing in both sides of $S^{d} V \otimes S^{\delta} V^{*} \otimes \wedge^{a} V \rightarrow S^{\delta} V \otimes \wedge^{a+1} V$

Note that if $S_{\pi} V \simeq S_{\mu} V$ as $S L(V)$-modules and the map is symmetric, then

$$
Y \operatorname{Flat}_{\pi, \mu}^{t}\left(S^{d} V\right)=\sigma_{t}\left(v_{2}\left(\mathbb{P} S_{\pi} V\right)\right) \cap \mathbb{P} S^{d} V,
$$

and if it is skew-symmetric, then

$$
Y F_{\text {Flat }}^{t}\left(S^{d} V\right)=\sigma_{t}\left(G\left(2, S_{\pi} V\right)\right) \cap \mathbb{P} S^{d} V .
$$

4.2. The surface case, $\sigma_{r}\left(v_{d}\left(\mathbb{P}^{2}\right)\right)$. In this subsection fix $\operatorname{dim} V=3$ and a volume form $\Omega$ on $V$. From the general formula for $\operatorname{dim} S_{\pi} V$ (see, e.g., [17, p78]), we record the special case:

$$
\operatorname{dim} S_{a, b} \mathbb{C}^{3}=\frac{1}{2}(a+2)(b+1)(a-b+1) .
$$

Lemma 4.2.1. Let $a \geq b$. Write $d=\alpha+\beta+\gamma$ with $\alpha \leq b, \beta \leq a-b$ so $S_{(a+\gamma-\alpha, b+\beta-\alpha)} V \subset$ $S_{a, b} V \otimes S^{d} V$. For $\phi \in S^{d} V$, consider the induced map

$$
\phi_{(a, b),(a+\gamma-\alpha, b+\beta-\alpha)}: S_{a, b} V^{*} \rightarrow S_{(a+\gamma-\alpha, b+\beta-\alpha)} V .
$$

Let $x \in V$, then

$$
\operatorname{rank}\left(\left(x^{d}\right)_{(a, b),(a+\gamma-\alpha, b+\beta-\alpha)}\right)=\frac{1}{2}(b-\alpha+1)(a-b-\beta+1)(a+\beta-\alpha+2)=: R .
$$

Thus in this situation $\wedge^{p R+1}\left(S_{a b} V\right) \otimes \wedge^{p R+1}\left(S_{a+\gamma-\alpha, b+\beta-\alpha} V\right)$ gives nontrivial degree $p R+1$ equations for $\sigma_{p}\left(v_{d}\left(\mathbb{P}^{2}\right)\right)$.

Remark 4.2.2. Note that the right hand sides of equations (8) and (10) are the same when $\alpha=\beta=0$. To get useful equations one wants $R$ small with respect to $\operatorname{dim} S_{a, b} \mathbb{C}^{3}$.

Proof. In the following picture we label the first row containing $a$ boxes with $a$ and so on.

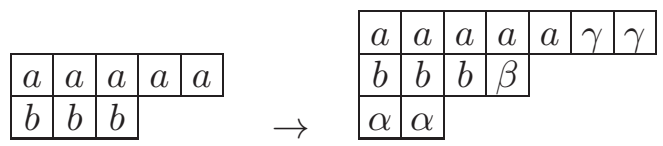

Assume we have chosen a weight basis $x_{1}, x_{2}, x_{3}$ of $V$ and $x=x_{3}$ is a vector of lowest weight. Consider the image of a weight basis of $S_{a, b} V$ under $\left(x_{3}^{3}\right)_{(a, b),(a+\gamma-\alpha, b+\beta-\alpha)}$. Namely consider all semi-standard fillings of the Young diagram corresponding to $(a, b)$, and count how many do not map to zero. By construction, the images of all the vectors that do not map to zero are linearly independent, so this count indeed gives the dimension of the image.

In order to have a vector not in the kernel, the first $\alpha$ boxes of the first row must be filled with 1's and the first $\alpha$ boxes of the second row must be filled with 2's.

Consider the next $(b-\alpha, b-\alpha)$ subdiagram. Let $\mathbb{C}_{i j}^{2}$ denote the span of $x_{i}, x_{j}$ and $\mathbb{C}_{i}^{1}$ the span of $x_{i}$. The boxes here can be filled with any semi-standard filling using 1's 2's and 3's, but the freedom to fill the rest will depend on the nature of the filling, so split $S_{b-\alpha, b-\alpha} \mathbb{C}^{3}$ into two parts, the first part where the entry in the last box in the first row is 1 , which has 
$\operatorname{dim} S_{b-\alpha} \mathbb{C}_{23}^{2}$ fillings and second where the entry in the last box in the first row is 2 , which has $\left(\operatorname{dim} S_{b-\alpha, b-\alpha} \mathbb{C}^{3}-\operatorname{dim} S_{b-\alpha} \mathbb{C}_{23}^{2}\right)$ fillings.

In the first case, the next $\beta$-entries are free to be any semi-standard row filled with 1's and 2's, of which there are $\operatorname{dim} S_{\beta} \mathbb{C}_{12}^{2}$ in number, but we split this further into two sub-cases, depending on whether the last entry is a 1 (of which there is one (= $\operatorname{dim} S_{\beta} \mathbb{C}_{1}^{1}$ ) such), or 2 (of which there are $\left(\operatorname{dim} S_{\beta} \mathbb{C}_{12}^{2}-\operatorname{dim} S_{\beta} \mathbb{C}_{1}^{1}\right)$ such). In the first sub-case the last $a-(b+\beta)$ entries admit $\operatorname{dim} S_{a-(b+\beta)} \mathbb{C}^{3}$ fillings and in the second sub-case there are $\operatorname{dim} S_{a-(b+\beta)} \mathbb{C}_{23}^{2}$ such. Putting these together, the total number of fillings for the various paths corresponding to the first part is

$$
\begin{aligned}
& \operatorname{dim} S_{b-\alpha} \mathbb{C}_{12}^{2}\left[\left(\operatorname{dim} S_{\beta} \mathbb{C}_{1}^{1}\right)\left(\operatorname{dim} S_{a-b-\beta} \mathbb{C}^{3}\right)+\left(\operatorname{dim} S_{\beta} \mathbb{C}_{12}^{2}-\operatorname{dim} S_{\beta} \mathbb{C}_{1}^{1}\right)\left(\operatorname{dim} S_{a-b-\beta} \mathbb{C}_{23}^{2}\right)\right] \\
& =(b-\alpha+1)\left[(1)\left(\begin{array}{c}
a-b-\beta+2 \\
2
\end{array}\right)+(\beta-1)(a-b-\beta+1)\right] .
\end{aligned}
$$

For the second part, the next $\beta$ boxes in the first row must be filled with 2's (giving $1=$ $\left.\operatorname{dim} S_{\beta} \mathbb{C}_{2}^{1}\right)$ and the last $a-(b+\beta)$ boxes can be filled with 2's or 3's semi-standardly, i.e., there are $\operatorname{dim} S_{a-b-\beta} \mathbb{C}_{23}^{2}$ 's worth. So the contribution of the second part is

$$
\begin{aligned}
& \left(\operatorname{dim} S_{b-\alpha, b-\alpha} \mathbb{C}^{3}-\operatorname{dim} S_{b-\alpha} \mathbb{C}_{12}^{2}\right)\left(\operatorname{dim} S_{\beta} \mathbb{C}_{2}^{1}\right)\left(\operatorname{dim} S_{a-b-\beta} \mathbb{C}_{23}^{2}\right) \\
& =\left(\left(\begin{array}{c}
b-\alpha+2 \\
b-\alpha
\end{array}\right)-(b-\alpha+1)\right)(1)(a-b-\beta+1) .
\end{aligned}
$$

Adding up gives the result.

We are particularly interested in cases where $(a, b)=(a+\gamma-\alpha, b+\beta-\alpha)$. In this case

$$
\begin{aligned}
& \alpha=\gamma=\frac{1}{3}(d+2 b-a) \\
& \beta=\frac{1}{3}(d-4 b+2 a) .
\end{aligned}
$$

Plugging into the conclusion of Lemma 4.2.1, the rank of the image of a $d$-th power in this situation is

$$
\frac{1}{9}(a+b-d+3)^{2}(a-b+1) .
$$

To keep this small, it is convenient to take $d=a+b$ so the rank is $a-b+1$. One can then fix this number and let $a, b$ grow to study series of cases.

If (10) has rank one when $d=2 p$, we just recover the usual symmetric flattenings as $S_{(p, p)} V=$ $S_{p} V^{*}$. We consider the next two cases in the theorems below, $(a, b)=(p+1, p)$ when $d=2 p+1$ and $(a, b)=(p+2, p)$ when $d=2 p+2$. Recall that $(p+q, p)^{*}=(p+q, q)$ in the notation of $\S 4.1 .1$.

Let $d=2 p+1$. The skew analog of Proposition 3.2.5 is the following proposition, which shows that the bound in the assumption of Theorem 1.2.3 is sharp.

Proposition 4.2.3. Let $\operatorname{dim} V=3$. If the variety $\sigma_{r}\left(v_{2 p+1}(\mathbb{P} V)\right)$ is an irreducible component of $Y$ Flat $_{(p+1, p),(p+1, p)}^{r}\left(S^{2 p+1} V\right)$, then $r \leq\left(\begin{array}{c}p+2 \\ 2\end{array}\right)$.

Proof. Recall from Proposition 2.4.4

$$
\begin{aligned}
\operatorname{codim} \sigma_{r}\left(v_{2 p+1}(\mathbb{P} V)\right) & =\left(\begin{array}{c}
2 p+3 \\
2
\end{array}\right)-3 r \\
\operatorname{codim} \sigma_{r}\left(G\left(2, S_{p+1, p} V\right) \cap \mathbb{P} S^{2 p+1} V\right. & \leq \frac{1}{2}[(p+1)(p+3)-2 r][(p+1)(p+3)-2 r-1]
\end{aligned}
$$

The inequality is a consequence of $\operatorname{codim} \sigma_{r}\left(v_{2 p+1}(\mathbb{P} V)\right) \leq \operatorname{codim} \sigma_{r}\left(G\left(2, S_{p+1, p} V\right) \cap \mathbb{P} S^{2 p+1} V\right.$. 
Here is a pictorial description when $p=2$ of $\phi_{31,31}: S_{32} V \rightarrow S_{31} V$ in terms of Young diagrams:

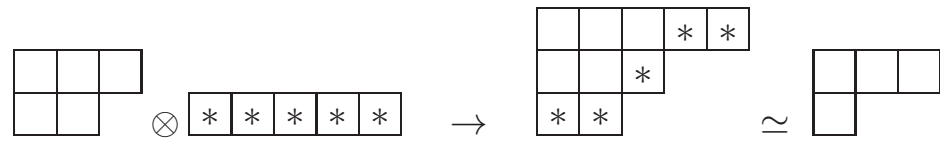

Corollary 4.2.4. $\operatorname{deg} \sigma_{\left(\begin{array}{c}p+2 \\ 2\end{array}\right)}\left(v_{2 p+1}\left(\mathbb{P}^{2}\right)\right) \leq \frac{1}{2^{p}} \prod_{i=0}^{p-1} \frac{\left(\begin{array}{c}(p+3)(p+1)+i \\ p-i\end{array}\right)}{\left(\begin{array}{c}2 i+1 \\ i\end{array}\right)}$.

If equality holds then $\sigma_{\left(\begin{array}{c}p+2 \\ 2\end{array}\right)}\left(v_{2 p+1}\left(\mathbb{P}^{2}\right)\right)$ is given scheme-theoretically by the size $(p+2)(p+$ 1) +2 sub-Pfaffians of $\phi_{(p+1, p),(p+1, p)}$.

The values of the right hand side for $p=1, \ldots 4$ are respectively $4,140,65780,563178924$.

Proof. The right hand side is $\operatorname{deg} \sigma_{\left(\begin{array}{c}p+2 \\ 2\end{array}\right)}\left(G\left(\mathbb{C}^{2}, S_{(p+1, p)} V\right)\right.$ by Proposition 2.4.4. Now $\sigma_{\left(\begin{array}{c}p+1 \\ 2\end{array}\right)}\left(v_{2 p+1}\left(\mathbb{P}^{2}\right)\right)$ is contained in a linear section of $\sigma_{\left(\begin{array}{c}p+2 \\ 2\end{array}\right)}\left(G\left(\mathbb{C}^{2}, S_{(p+1, p)} V\right)\right)$, and by Theorem 1.2.3 it is a irreducible component of this linear section. The result follows by the refined Bezout theorem 2.4.1.

In Corollary 4.2.4 equality holds in the cases $p=1,2$ by Proposition 2.4.2. The case $p=1$ is just the Aronhold case and the case $p=2$ will be considered in Theorem 4.2.7 (2). For $p \geq 3$ these numbers are out of the range of [15] and we do not know if equality holds.

Note that the usual symmetric flattenings only give equations for $\sigma_{k-1}\left(v_{2 p+1}\left(\mathbb{P}^{2}\right)\right)$ for $k \leq$ $\frac{1}{2}\left(p^{2}+3 p+2\right)$.

Now let $d=2 p+2$ be even, requiring $\pi=\mu$, the smallest possible $\operatorname{rank}\left(\left(x^{d}\right)_{\pi, \mu}\right)$ is three, which we obtain with $\phi_{(p+2, p),(p+2, p)}$.

Proposition 4.2.5. Let $d=2 p+2$. The Young flattening $\phi_{(p+2,2),(p+2,2)} \in S_{p+2,2} V \otimes S_{p+2,2} V$ is symmetric. It is of rank three for $\phi \in v_{d}\left(\mathbb{P}^{2}\right)$ and gives degree $3(k+1)$ equations for $\sigma_{r}\left(v_{2 p+2}\left(\mathbb{P}^{2}\right)\right)$ for $r \leq \frac{1}{2}\left(p^{2}+5 p+4\right)-1$. A convenient model for the equations is given in the proof.

A pictorial description when $p=2$ is as follows:

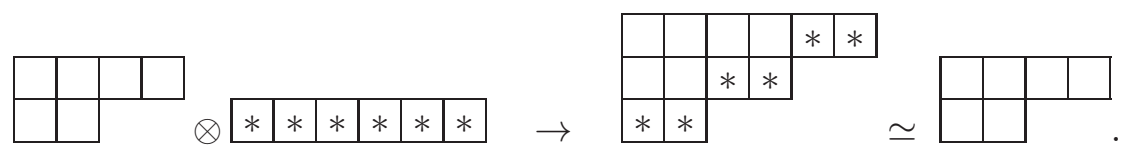

Proof. Let $\tilde{\Omega} \in \wedge^{3} V$ be dual to the volume form $\Omega$. To prove the symmetry, for $\phi=x^{2 p+2}$, consider the map,

$$
\begin{aligned}
M_{x^{2 p+2}}: S^{p} V^{*} \otimes S^{2}\left(\wedge^{2} V^{*}\right) & \rightarrow S^{p} V \otimes S^{2}\left(\wedge^{2} V\right) \\
\alpha_{1} \cdots \alpha_{p} \otimes\left(\gamma_{1} \wedge \delta_{1}\right) \circ\left(\gamma_{2} \wedge \delta_{2}\right) & \left.\left.\mapsto \alpha_{1}(x) \cdots \alpha_{p}(x) x^{p} \otimes \tilde{\Omega}(x\lrcorner \gamma_{1} \wedge \delta_{1}\right) \circ \tilde{\Omega}(x\lrcorner \gamma_{2} \wedge \delta_{2}\right)
\end{aligned}
$$

and define $M_{\phi}$ for arbitrary $\phi \in S^{2 p+2} V$ by linearity and polarization. If we take bases of $S^{2} V \otimes S^{2}\left(\wedge^{2} V\right)$ as above, with indices $\left(\left(i_{1}, \ldots, i_{p}\right),(k l),\left(k^{\prime} l^{\prime}\right)\right)$, most of the matrix of $M_{e_{1}^{2 p+2}}$ is zero. The upper-right hand $6 \times 6$ block, where $\left(i_{1}, \ldots, i_{p}\right)=(1, \ldots, 1)$ in both rows and columns and the order on the other indices

$$
\text { ((12), (12)), ((13), (13)), ((12), (13)), ((12), (23)), ((13), (23)), ((23), (23)), }
$$


is

showing the symmetry. Now

$$
\left(\begin{array}{llllll}
0 & 1 & 0 & 0 & 0 & 0 \\
1 & 0 & 0 & 0 & 0 & 0 \\
0 & 0 & 1 & 0 & 0 & 0 \\
0 & 0 & 0 & 0 & 0 & 0 \\
0 & 0 & 0 & 0 & 0 & 0 \\
0 & 0 & 0 & 0 & 0 & 0
\end{array}\right)
$$

$$
\begin{aligned}
\left(S^{p} V \otimes S^{2}\left(\wedge^{2} V\right)\right)^{\otimes 2} & =\left(S^{p} V \otimes\left(S_{22} V \oplus S_{1111} V\right)^{\otimes 2}\right. \\
& =S_{p+2,2} V \oplus \text { stuff }
\end{aligned}
$$

where all the terms in stuff have partitions with at least three parts. On the other hand, from the nature of the image we conclude it is just the first factor and $M_{\phi} \in S^{2}\left(S_{p+2,2} V\right)$.

Note that the usual symmetric flattenings give nontrivial equations for $\sigma_{k-1}\left(v_{2 p+2}\left(\mathbb{P}^{2}\right)\right)$ for $k \leq \frac{1}{2}\left(p^{2}+5 p+6\right)$, a larger range than in Proposition 4.2.5. However we show (Theorem 4.2.9) that the symmetric flattenings alone are not enough to cut out $\sigma_{7}\left(v_{6}\left(\mathbb{P}^{2}\right)\right)$, but with the $((p+1,2),(p+1,2))$-Young flattening they are.

Here is a more general Young flattening:

Proposition 4.2.6. Let $d=p+4 q-1$. The Young flattening

$$
\phi_{(p+2 q, 2 q-1),(p+2 q, 2 q-1)} \in S_{(p+2 q, 2 q-1)} V \otimes S_{(p+2 q, 2 q-1)} V,
$$

is skew-symmetric if $p$ is even and symmetric if $p$ is odd.

Since it has rank $p$ if $\phi \in v_{d}\left(\mathbb{P}^{2}\right)$, if $p$ is even (resp. odd), the size $k p+2$ sub-Pfaffians (resp. size $k p+1$ minors $)$ of $\phi_{(p+2 q, 2 q-1),(p+2 q, 2 q-1)}$ give degree $\frac{k p}{2}+1$ (resp. $\left.k p+1\right)$ equations for $\sigma_{k}\left(v_{p+4 q-1}\left(\mathbb{P}^{2}\right)\right)$ for

$$
k \leq \frac{q(p+2 q+2)(p+2)}{p} .
$$

Proof. Consider $M_{\phi}: S^{p-1} V^{*} \otimes S^{q}\left(\wedge^{2} V^{*}\right) \rightarrow S^{p} V \otimes S^{q}\left(\wedge^{2} V\right)$ given for $\phi=x^{p+4 q-1}$ by

$$
\left.\left.\alpha_{1} \cdots \alpha_{p-1} \otimes \beta_{1} \wedge \gamma_{1} \cdots \beta_{q} \wedge \gamma_{q} \mapsto \Pi_{j}\left(\alpha_{j}(x)\right) x^{p-1} \otimes \tilde{\Omega}(x\lrcorner \beta_{1} \wedge \gamma_{1}\right) \cdots \tilde{\Omega}(x\lrcorner \beta_{q} \wedge \gamma_{q}\right)
$$

and argue as above.

Here the usual flattenings give degree $k$ equations for $\sigma_{k-1}\left(v_{d}\left(\mathbb{P}^{2}\right)\right)$ in the generally larger range $k \leq \frac{1}{8}(p+4 q+2)(p+4 q)$.

Recall that we have already determined the ideals of $\sigma_{r}\left(v_{d}\left(\mathbb{P}^{2}\right)\right)$ for $d \leq 4$, (Thm. 3.2.1 and the chart in the introduction) so we next consider the case $d=5$.

Case $d=5$ : The symmetric flattening given by the size $(k+1)$ minors of $\phi_{2,3}$ define $\sigma_{k}\left(v_{5}\left(\mathbb{P}^{2}\right)\right)$ up to $k=4$ by Theorem 3.1.4 (3). Note that the size 6 minors define a subvariety of codimension 5 , strictly containing $\sigma_{5}\left(v_{5}\left(\mathbb{P}^{2}\right)\right)$, which has codimension 6 . So, in this case, the bound provided by Theorem 3.1 .4 is sharp.

\section{Theorem 4.2.7.}

(1) $\sigma_{k}\left(v_{5}\left(\mathbb{P}^{2}\right)\right)$ for $k \leq 5$ is an irreducible component of $Y$ Flat ${ }_{31,31}^{2 k}\left(S^{5} \mathbb{C}^{3}\right)$, the variety given by the principal size $2 k+2$ Pfaffians of the [(31),(31)]-Young flattenings.

(2) the principal size 14 Pfaffians of the [(31),(31)]-Young flattenings are scheme-theoretic defining equations for $\sigma_{6}\left(v_{5}\left(\mathbb{P}^{2}\right)\right)$, i.e., as schemes, $\sigma_{6}\left(v_{5}\left(\mathbb{P}^{2}\right)\right)=$ Y Flat ${ }_{31,31}^{12}\left(S^{5} \mathbb{C}^{3}\right)$.

(3) $\sigma_{7}\left(v_{5}\left(\mathbb{P}^{2}\right)\right)$ is the ambient space. 
Proof. (1) is a consequence of Theorem 1.2.3.

To prove the second assertion, by the Segre formula (Proposition 2.4.4) the subvariety of size 15 skew-symmetric matrices of rank $\leq 12$ has codimension 3 and degree 140. By Proposition 2.4.2, $\sigma_{6}\left(v_{5}\left(\mathbb{P}^{2}\right)\right)$ has codimension 3 and degree 140 . We conclude as in the proof of Corollary 3.2 .6 .

Case $d=6$ : In the case $\sigma_{k}\left(v_{6}\left(\mathbb{P}^{2}\right)\right)$, the symmetric flattening given by the $(k+1)$ minors of $\phi_{2,4}$ define $\sigma_{k}\left(v_{6}\left(\mathbb{P}^{2}\right)\right)$ as an irreducible component up to $k=4$ (in the case $k=4 \mathrm{~S}$. Diesel [25, Ex. 3.6] showed that there are two irreducible components) and the symmetric flattening given by the $(k+1)$ minors of $\phi_{3,3}$ define $\sigma_{k}\left(v_{6}\left(\mathbb{P}^{2}\right)\right)$ as an irreducible component up to $k=6$.

The following is a special case of the Thm. 3.2.1(4), we include this second proof because it is very short.

Theorem 4.2.8. As schemes, $\sigma_{6}\left(v_{6}\left(\mathbb{P}^{2}\right)\right)=\operatorname{Rank}_{3,3}^{6}\left(S^{6} \mathbb{C}^{3}\right)$, i.e., the size 7 minors of $\phi_{3,3}$ cut out $\sigma_{6}\left(v_{6}\left(\mathbb{P}^{2}\right)\right)$ scheme-theoretically.

Proof. By the Segre formula (Proposition 2.4.4) the subvariety of symmetric $10 \times 10$ matrices of rank $\leq 6$ has codimension 10 and degree 28,314. By Proposition 2.4.2, $\sigma_{6}\left(v_{6}\left(\mathbb{P}^{2}\right)\right)$ has codimension 10 and degree 28,314. We conclude as in the proof of Corollary 3.2.6.

The size 8 minors of $\phi_{3,3}$ define a subvariety of codimension 6 , strictly containing $\sigma_{7}\left(v_{6}\left(\mathbb{P}^{2}\right)\right)$, which has codimension 7 . In the same way, the size 9 minors of $\phi_{3,3}$ define a subvariety of codimension 3 , strictly containing $\sigma_{8}\left(v_{6}\left(\mathbb{P}^{2}\right)\right)$, which has codimension 4 . Below we construct equations in terms of Young flattenings.

$\operatorname{det}\left(\phi_{42,42}\right)$ is a polynomial of degree 27 , which is not the power of a lower degree polynomial. This can be proved by cutting with a random projective line, and using Macaulay2. The two variable polynomial obtained is not the power of a lower degree polynomial.

If $\phi$ is decomposable then $\operatorname{rank}\left(\phi_{42,42}\right)=3$ by Lemma 4.2.1, so that when $\phi \in \sigma_{k}\left(v_{6}\left(\mathbb{P}^{2}\right)\right)$ then $\operatorname{rank}\left(\phi_{42,42}\right) \leq 3 k$.

\section{Theorem 4.2.9.}

(1) $\sigma_{7}\left(v_{6}\left(\mathbb{P}^{2}\right)\right)$ is an irreducible component of $\operatorname{Rank}_{3,3}^{7}\left(S^{6} \mathbb{C}^{3}\right) \cap Y$ Flat ${ }_{42,42}^{22}\left(S^{6} \mathbb{C}^{3}\right)$, i.e., of the variety defined by the size 8 minors of the symmetric flattening $\phi_{3,3}$ and by the size 22 minors of the $[(42),(42)]$-Young flattenings.

(2) $\sigma_{8}\left(v_{6}\left(\mathbb{P}^{2}\right)\right)$ is an irreducible component of $\operatorname{Rank}_{3,3}^{8}\left(S^{6} \mathbb{C}^{3}\right) \cap Y$ Flat $_{42,42}^{24}\left(S^{6} \mathbb{C}^{3}\right)$, i.e., of the variety defined by the size 9 minors of the symmetric flattening $\phi_{3,3}$ and by the size 25 minors of the [(42), (42)]-Young flattenings.

(3) $\sigma_{9}\left(v_{6}\left(\mathbb{P}^{2}\right)\right)$ is the hypersurface of degree 10 defined by $\operatorname{det}\left(\phi_{3,3}\right)$.

Proof. To prove (2), we picked a polynomial $\phi$ which is the sum of 8 random fifth powers of linear forms, and a submatrix of $\phi_{42,42}$ of order 24 which is invertible. The matrix representing $\phi_{42,42}$ can be constructed explicitly by the package PieriMaps of Macaulay2 [22, 45].

The affine tangent space at $\phi$ of $\operatorname{Rank}_{3,3}^{8}\left(S^{6} \mathbb{C}^{3}\right)$ has codimension 3 in $S^{6} \mathbb{C}^{3}$. In order to compute a tangent space, differentiate, as usual, each line of the matrix, substitute $\phi$ in the other lines, compute the determinant and then sum over the lines. It is enough to pick one minor of order 25 of $\phi_{42,42}$ containing the invertible ones of order 24 . The tangent space of this minor at $\phi$ is not contained in the subspace of codimension 3, yielding the desired subspace of codimension 4.

(1) can be proved in the same way. (3) is well known.

Remark 4.2.10. $\sigma_{7}\left(v_{6}\left(\mathbb{P}^{2}\right)\right)$ is the first example where the known equations are not of minimal possible degree. 
Case $d=7$ : In the case of $\sigma_{k}\left(v_{7}\left(\mathbb{P}^{2}\right)\right)$, the symmetric flattening given by the size $(k+1)$ minors of $\phi_{3,4}$ define $\sigma_{k}\left(v_{7}\left(\mathbb{P}^{2}\right)\right)$ as an irreducible component up to $k=8$.

\section{Theorem 4.2.11.}

(1) For $k \leq 10 \sigma_{k}\left(v_{7}\left(\mathbb{P}^{2}\right)\right)$ is an irreducible component of $Y$ Flat $t_{41,41}^{k}\left(S^{7} \mathbb{C}^{3}\right)$, which is defined by the size $(2 k+2)$ subpfaffians of of $\phi_{41,41}$.

(2) $\sigma_{11}\left(v_{7}\left(\mathbb{P}^{2}\right)\right)$ has codimension 3 and it is contained in the hypersurface Y Flat ${ }_{41,41}^{22}\left(S^{7} \mathbb{C}^{3}\right)$ of degree 12 defined by $\operatorname{Pf}\left(\phi_{41,41}\right)$.

Proof. (1) follows from Theorem 1.2.3. (2) is obvious.

Remark 4.2.12. We emphasize that $\sigma_{11}\left(v_{7}\left(\mathbb{P}^{2}\right)\right)$ is the first case where we do not know, even conjecturally, further equations.

Case $d=8$ : It is possible that $\sigma_{k}\left(v_{8}\left(\mathbb{P}^{2}\right)\right)$ is an irreducible component of $\operatorname{Rank}_{4,4}^{k+1}\left(S^{8} \mathbb{C}^{3}\right)$ up to $k=12$, but the bound given in Theorem 3.1.4 is just $k \leq 10$. The size 14 minors of $\phi_{4,4}$ define a subvariety of codimension 4 , which strictly contains $\sigma_{13}\left(v_{8}\left(\mathbb{P}^{2}\right)\right)$ which has codimension 6. Other equations of degree 40 for $\sigma_{13}\left(v_{8}\left(\mathbb{P}^{2}\right)\right)$ are given by the size 40 minors of $\phi_{53,52}$. It is possible that minors of $\phi_{(53),(52)}$ could be used to get a collection of set-theoretic equations for $\sigma_{12}\left(v_{8}\left(\mathbb{P}^{2}\right)\right)$ and $\sigma_{11}\left(v_{8}\left(\mathbb{P}^{2}\right)\right)$. In the same way, $\operatorname{det} \phi_{4,4}$ is just an equation of degree 15 for $\sigma_{14}\left(v_{8}\left(\mathbb{P}^{2}\right)\right)$ which has codimension 3 .

Case $d=9$ : It is possible that $\sigma_{k}\left(v_{9}\left(\mathbb{P}^{2}\right)\right)$ is an irreducible component of $\operatorname{Ran} k_{4,5}^{k+1}\left(S^{9} \mathbb{C}^{3}\right)$ up to $k=13$, but the bound given in Theorem 3.1.4 is just $k \leq 11$. $\operatorname{Ran}_{4,5}^{14}\left(S^{9} \mathbb{C}^{3}\right)$ strictly contains $\sigma_{14}\left(v_{9}\left(\mathbb{P}^{2}\right)\right)$ which has codimension 13 . The principal Pfaffians of order 30 of $\phi_{54,51}$ give further equations for $\sigma_{14}\left(v_{9}\left(\mathbb{P}^{2}\right)\right)$. In the same way the principal Pfaffians of order 32 (resp. 34) of $\phi_{54,51}$ give some equations for $\sigma_{15}\left(v_{9}\left(\mathbb{P}^{2}\right)\right)$ (resp. $\left.\sigma_{16}\left(v_{9}\left(\mathbb{P}^{2}\right)\right)\right)$. Another equation for $\sigma_{15}\left(v_{9}\left(\mathbb{P}^{2}\right)\right)$ is the Pfaffian of the skew-symmetric morphism $\phi_{63,63}: S_{6,3} V \rightarrow S_{6,3} V$ which can be pictorially described in terms of Young diagrams, by
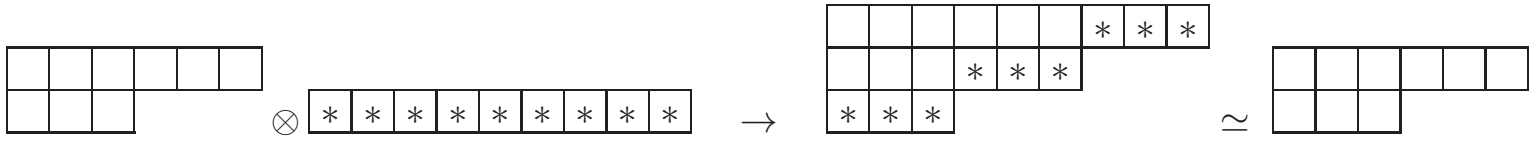

We do not know any equations for $\sigma_{k}\left(v_{9}\left(\mathbb{P}^{2}\right)\right)$ when $k=17,18$. The $k=18$ case is particularly interesting because the corresponding secant variety is a hypersurface.

\section{Construction of equations from vector Bundles}

5.1. Main observation. Write $e:=\operatorname{rank}(E)$ and consider the determinantal varieties or rank varieties defined by the minors of $A_{v}^{E}: H^{0}(E) \rightarrow H^{0}\left(E^{*} \otimes L\right)^{*}$ (defined by equation (3)),

$$
\begin{aligned}
\operatorname{Rank}_{k}(E): & =\mathbb{P}\left\{v \in V \mid \operatorname{rank}\left(A_{v}^{E}\right) \leq e k\right\} \\
& =\sigma_{e k}\left(\operatorname{Seg}\left(\mathbb{P} H^{0}(E)^{*} \times \mathbb{P} H^{0}\left(E^{*} \otimes L\right)^{*}\right)\right) \cap \mathbb{P} V .
\end{aligned}
$$

Proposition 5.1.1. Let $X \subset \mathbb{P} V=\mathbb{P} H^{0}(L)^{*}$ be a variety, and $E$ a rank $e$ vector bundle on $X$. Then

$$
\sigma_{r}(X) \subseteq \operatorname{Rank}_{r}(E)
$$

i.e., the size $(r e+1)$ minors of $A_{v}^{E}$ give equations for $\sigma_{r}(X)$.

When $E$ is understood, we will write $A_{v}$ for $A_{v}^{E}$.

Proof. By (3), if $x=[v] \in X$, then $H^{0}\left(I_{x} \otimes E\right) \subseteq \operatorname{ker} A_{v}$. The subspace $H^{0}\left(I_{x} \otimes E\right) \subseteq H^{0}(E)$ has codimension at most $e$ in $H^{0}(E)$, hence the same is true for the subspace $\operatorname{ker} A_{x}$ and it 
follows $\operatorname{rank}\left(A_{x}\right) \leq e$. If $v \in \hat{\sigma}_{r}(X)$ is a general point, then it may be expressed as $v=\sum_{i=1}^{r} x_{i}$, with $x_{i} \in \hat{X}$. Hence

$$
\operatorname{rank}\left(A_{v}\right)=\operatorname{rank}\left(\sum_{i=1}^{r} A_{x_{i}}\right) \leq \sum_{i=1}^{r} \operatorname{rank}\left(A_{x_{i}}\right) \leq r e
$$

as claimed. Since the inequality is a closed condition, it holds for all $v \in \hat{\sigma}_{r}(X)$.

Example 5.1.2. Let $X=v_{d}(\mathbb{P} W), L=\mathcal{O}(d), E=\mathcal{O}(a)$ so $E^{*} \otimes L=\mathcal{O}(d-a)$, then $\operatorname{Rank}_{r}(E)$ is the catalecticant variety of symmetric flattenings in $S^{a} W \otimes S^{d-a} W$ of rank at most $r$.

Example 5.1.3. Let $E$ be a homogeneous bundle on $\mathbb{P}^{n}$ with $H^{0}(E)^{*}=S_{\mu} V, H^{0}\left(E^{*} \otimes L\right)^{*}=$ $S_{\pi} V$. Then $A_{v}$ corresponds to $\phi_{\pi, \mu}$ of $\S 4$.

The generalization of symmetric flattenings to Young flattenings for Veronese varieties is a representation-theoretic version of the generalization from line bundles to higher rank vector bundles.

Example 5.1.4. A general source of examples is given by curves obtained as determinantal loci. This topic is studied in detail in [14]. In [21], A. Ginensky considers the secant varieties $\sigma_{k}(C)$ to smooth curves $C$ in their bicanonical embedding. With our notations this corresponds to the symmetric pair $(E, L)=\left(K_{C}, K_{C}^{2}\right)$. He proves [21, Thm. 2.1] that $\sigma_{k}(C)=\operatorname{Rank}_{k}\left(K_{C}\right)$ if $k<\operatorname{Cliff}(C)$ and $\sigma_{k}(C) \subsetneq \operatorname{Rank}_{k}\left(K_{C}\right)$ for larger $k$. Here Cliff $(C)$ denotes the Clifford index of $C$.

5.2. The construction in the symmetric and skew-symmetric cases. We say that $(E, L)$ is a symmetric pair if the isomorphism $E \stackrel{\alpha}{\longrightarrow} E^{*} \otimes L$ is symmetric, that is the transpose isomorphism $E \otimes L^{*} \stackrel{\alpha^{t}}{\longrightarrow} E^{*}$, after tensoring by $L$ and multiplying the map by $1_{L}$ equals $\alpha$, i.e., $\alpha=\alpha^{t} \otimes 1_{L}$. In this case $S^{2} E$ contains $L$ as a direct summand, the morphism $A_{v}$ is symmetric, and $\operatorname{Rank}_{k}(E)$ is defined by the $(k e+1)$-minors of $A_{v}$.

Similarly, $(E, L)$ is a skew-symmetric pair if $\alpha=-\alpha^{t} \otimes 1_{L}$. In this case $e$ is even, $\wedge^{2} E$ contains $L$ as a direct summand, the morphism $A_{v}$ is skew-symmetric, and $\operatorname{Rank}_{k}(E)$ is defined by the size $(k e+2)$ subpfaffians of $A_{v}$, which are equations of degree $\frac{k e}{2}+1$.

Example 5.2.1. Let $X=\mathbb{P}^{2} \times \mathbb{P}^{n}$ embedded by $L=\mathcal{O}(1,2)$. The equations for $\sigma_{k}(X)$ recently considered in [8], where they have been called exterior flattenings, fit in this setting. Call $p_{1}, p_{2}$ the two projections. Let $Q$ be the tautological quotient bundle on $\mathbb{P}^{2}$ and let $E=p_{1}^{*} Q \otimes p_{2}^{*} \mathcal{O}(1)$, then $(E, L)$ is a skew-symmetric pair which gives rise to the equations (2) in Theorem 1.1 of [8], while the equations (1) are obtained with $E=p_{2}^{*} \mathcal{O}(1)$.

5.3. The conormal space. The results reviewed in $\S 2.5$, restated in the language of vector bundles, say the affine conormal space of $\operatorname{Rank}_{k}(E)$ at $[v] \in \operatorname{Rank}_{k}(E)_{\text {smooth }}$ is the image of the map

$$
\operatorname{ker} A_{v} \otimes \operatorname{Im} A_{v}^{\perp} \rightarrow H^{0}(L)=V^{*} .
$$

If $(E, L)$ is a symmetric (resp. skew symmetric) pair, there is a symmetric (resp. skew symmetric) isomorphism ker $A_{v} \simeq \operatorname{Im} A_{v}^{\perp}$ and the conormal space of $\operatorname{Rank}_{k}(E)$ at $v$ is given by the image of the map $S^{2}\left(\operatorname{ker} A_{v}\right) \rightarrow H^{0}(L),\left(\operatorname{resp} . \wedge^{2}\left(\operatorname{ker} A_{v}\right) \rightarrow H^{0}(L)\right)$.

5.4. A sufficient criterion for $\sigma_{k}(X)$ to be an irreducible component of $\operatorname{Rank}_{k}(E)$. Let $v \in \hat{X}$, in the proof of Proposition 5.1.1, we saw $H^{0}\left(I_{v} \otimes E\right) \subseteq \operatorname{ker} A_{v}$. In the same way, $H^{0}\left(I_{v} \otimes E^{*} \otimes L\right) \subseteq \operatorname{Im} A_{v}^{\perp}$, by taking transpose. Equality holds if $E$ is spanned at $x=[v]$. This is generalized by the following Proposition. 
Proposition 5.4.1. Let $v=\sum_{i=1}^{k} x_{i} \in V$, with $\left[x_{i}\right] \in X$, and let $Z=\left\{\left[x_{1}\right], \ldots,\left[x_{k}\right]\right\}$. Then

$$
\begin{aligned}
H^{0}\left(I_{Z} \otimes E\right) & \subseteq \operatorname{ker} A_{v} \\
H^{0}\left(I_{Z} \otimes E^{*} \otimes L\right) & \subseteq \operatorname{Im} A_{v}^{\perp} .
\end{aligned}
$$

The first inclusion is an equality if $H^{0}\left(E^{*} \otimes L\right) \rightarrow H^{0}\left(E^{*} \otimes L_{\mid Z}\right)$ is surjective. The second inclusion is an equality if $H^{0}(E) \rightarrow H^{0}\left(E_{\mid Z}\right)$ is surjective.

Remark 5.4.2. In [3], a line bundle $F \rightarrow X$ is defined to be $k$-spanned if $H^{0}(F)$ surjects onto $H^{0}\left(\left.F\right|_{Z}\right)$ for all $Z=\left\{\left[x_{1}\right], \ldots,\left[x_{k}\right]\right\}$. In that paper and in subsequent work they study which line bundles have this property. In particular $k$-spanned-ness of $X \subset \mathbb{P} H^{0}(F)^{*}$ implies that for all $k$-tuples of points $\left(\left[x_{1}\right], \ldots,\left[x_{k}\right]\right)$ on $X$, writing $Z=\left\{\left[x_{1}\right], \ldots,\left[x_{k}\right]\right\}$, then $\langle Z\rangle=\mathbb{P}^{k-1}$, as for example occurs with Veronese varieties $v_{d}(\mathbb{P} V)$ when $k \leq d+1$.

Proof. If $E^{*} \otimes L$ is spanned at $x=[w]$ then we claim $H^{0}\left(I_{x} \otimes E\right)=\operatorname{ker} A_{w}$. To see this, work over an open set where $E, L$ are trivializable and take trivializations. There are $t_{j} \in H^{0}\left(E^{*} \otimes L\right)$ such that in a basis $e_{i}$ of the $\mathbb{C}^{e}$ which we identify with the fibers of $E$ on this open subset, $\left\langle e_{i}, t_{j}(x)\right\rangle=\delta_{i j}$. Take $s \in \operatorname{ker}\left(A_{w}\right)$. By assumption $\left\langle s(x), t_{j}(x)\right\rangle=0$ for every $j$, hence, writing $s=\sum s_{i} e_{i}, s_{j}(x)=0$ for every $j$, i.e., $s \in H^{0}\left(I_{x} \otimes E\right)$.

Since

$$
H^{0}\left(I_{Z} \otimes E\right)=\cap_{i=1}^{k} H^{0}\left(I_{x_{i}} \otimes E\right) \subseteq \cap_{i=1}^{k} \operatorname{ker} A_{x_{i}} \subseteq \operatorname{ker} A_{v},
$$

the inclusion for the kernel follows. To see the equality assertion, if $H^{0}\left(E^{*} \otimes L\right) \rightarrow H^{0}\left(E^{*} \otimes L_{\mid Z}\right)$ is surjective, for every $j=1, \ldots, k$ we can choose $t_{h, j} \in H^{0}\left(E^{*} \otimes L\right)$, for $h=1, \ldots, e$, such that $t_{h, j}\left(x_{i}\right)=0$ for $i \neq j, \forall h$ and $t_{h, j}$ span the fiber of $E^{*} \otimes L$ at $x_{j}$. It follows that if $s \in \operatorname{ker}\left(A_{v}\right)$ then $t_{h, j}\left(x_{j}\right) \cdot s\left(x_{j}\right)=0$ for every $h, j$ which implies $s\left(x_{j}\right)=0$, that is $s \in H^{0}\left(I_{Z} \otimes E\right)$. The dual statement is similar.

The following theorem gives a useful criterion to find local equations of secant varieties.

Theorem 5.4.3. Let $v=\sum_{i=1}^{r} x_{i} \in V$ and let $Z=\left\{\left[x_{1}\right], \ldots,\left[x_{r}\right]\right\}$, where $\left[x_{j}\right] \in X$. If

$$
H^{0}\left(I_{Z} \otimes E\right) \otimes H^{0}\left(I_{Z} \otimes E^{*} \otimes L\right) \longrightarrow H^{0}\left(I_{Z^{2}} \otimes L\right)
$$

is surjective, then $\sigma_{r}(X)$ is an irreducible component of $\operatorname{Rank}_{r}(E)$.

If $(E, L)$ is a symmetric, resp. skew-symmetric pair, and

$$
\begin{aligned}
S^{2}\left(H^{0}\left(I_{Z} \otimes E\right)\right) & \rightarrow H^{0}\left(I_{Z^{2}} \otimes L\right) \\
\text { resp. } \quad \wedge^{2}\left(H^{0}\left(I_{Z} \otimes E\right)\right) & \rightarrow H^{0}\left(I_{Z^{2}} \otimes L\right)
\end{aligned}
$$

is surjective, then $\sigma_{r}(X)$ is an irreducible component of $\operatorname{Rank}_{r}(E)$.

Proof. Write $v=x_{1}+\cdots+x_{r}$ for a smooth point of $\hat{\sigma}_{r}(X)$. Recall that by Terracini's Lemma, $\mathbb{P} \hat{N}_{[v]}^{*} \sigma_{r}(X)$ is the space of hyperplanes $H \in \mathbb{P} V^{*}$ such that $H \cap \sigma_{r}(X)$ is singular at the $\left[x_{i}\right]$, i.e., $\hat{N}_{[v]}^{*} \sigma_{r}(X)=H^{0}\left(I_{Z^{2}} \otimes L\right)$. Consider the commutative diagram

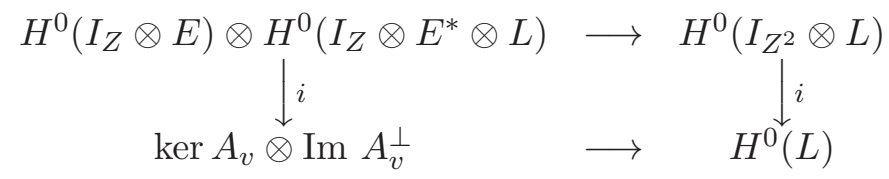

The surjectivity of the map in the first row implies that the rank of map in the second row is at least $\operatorname{dim} H^{0}\left(I_{Z^{2}} \otimes L\right)$. By Proposition 5.4.1, ker $A_{v} \otimes \operatorname{Im} A_{v}^{\perp} \rightarrow H^{0}\left(I_{Z^{2}} \otimes L\right)$ is surjective, so that the conormal spaces of $\sigma_{k}(X)$ and of $\operatorname{Rank}_{k}(E)$ coincide at $v$, proving the general case. The symmetric and skew-symmetric cases are analogous. 


\section{The induction Lemma}

6.1. Weak defectivity. The notion of weak defectivity, which dates back to Terracini, was applied by Ciliberto and Chiantini in [10] to show many cases of tensors and symmetric tensors admitted a unique decomposition as a sum of rank one tensors. We review here it as it is used to prove the promised induction Lemma 6.2.1.

Definition 6.1.1. [10, Def. 1.2] A projective variety $X \subset \mathbb{P} V$ is $k$-weakly defective if the general hyperplane tangent in $k$ general points of $X$ exists and is tangent along a variety of positive dimension.

Notational warning: what we call $k$-weakly defective is called $(k-1)$-weakly defective in [10]. We shifted the index in order to uniformize to our notion of $k$-defectivity: by Terracini's lemma, $k$-defective varieties (i.e., those where $\operatorname{dim} \sigma_{k}(X)$ is less than the expected dimension) are also $k$-weakly defective.

The Veronese varieties which are weakly defective have been classified.

Theorem 6.1.2 (Chiantini-Ciliberto-Mella-Ballico). [10, 36, 1] The $k$-weakly defective varieties $v_{d}\left(\mathbb{P}^{n}\right)$ are the triples $(k, d, n)$ :

(i) the $k$-defective varieties, namely $(k, 2, n), k=2, \ldots,\left(\begin{array}{c}n+2 \\ 2\end{array}\right),(5,4,2),(9,4,3),(14,4,4)$, $(7,3,4)$,

and

(ii) $(9,6,2),(8,4,3)$.

Let $L$ be an ample line bundle on a variety $X$. Recall that the discriminant variety of a subspace $V \subseteq H^{0}(L)$ is given by the elements of $\mathbb{P} V$ whose zero sets (as sections of $L$ ) are singular outside the base locus of common zeros of elements of $V$. When $V$ gives an embedding of $X$, then the discriminant variety coincides with the dual variety of $X \subset \mathbb{P} V^{*}$.

Proposition 6.1.3. Assume that $X \subset \mathbb{P} V=\mathbb{P} H^{0}(L)^{*}$ is not $k$-weakly defective and that $\sigma_{k}(X)$ is a proper subvariety of $\mathbb{P} V$. Then for a general $Z^{\prime}$ of length $k^{\prime}<k$, the discriminant subvariety in $\mathbb{P} H^{0}\left(I_{Z^{\prime 2}} \otimes L\right)$, given by the hyperplane sections having an additional singular point outside $Z^{\prime}$, is not contained in any hyperplane.

Proof. If $X$ is not $k$-weakly defective, then it is not $k^{\prime}$-weakly defective for any $k^{\prime} \leq k$. Hence we may assume $k^{\prime}=k-1$. Consider the projection $\pi$ of $X$ centered at the span of $k^{\prime}$ general tangent spaces at $X$. The image $\pi(X)$ is not 1-weakly defective, [10, Prop 3.6] which means that the Gauss map of $\pi(X)$ is nondegenerate [10, Rem. 3.1 (ii)]. Consider the dual variety of $\pi(X)$, which is contained in the discriminant, see [34, p. 810]. If the dual variety of $\pi(X)$ were contained in a hyperplane, then $\pi(X)$ would be a cone (see, e.g. [13, Prop. 1.1]), and thus be 1 -weakly defective, which is a contradiction. Hence the linear span of the discriminant variety is the ambient space.

6.2. If $X$ is not $k$-weakly defective and the criterion of $\S 5.4$ works for $k$, it works for $k^{\prime} \leq k$.

Lemma 6.2.1. Let $X \subset \mathbb{P} V=\mathbb{P} H^{0}(L)^{*}$ be a variety and $E \rightarrow X$ be a vector bundle on $X$. Assume that

$$
H^{0}\left(I_{Z} \otimes E\right) \otimes H^{0}\left(I_{Z} \otimes E^{*} \otimes L\right) \longrightarrow H^{0}\left(I_{Z^{2}} \otimes L\right)
$$

is surjective for the general $Z$ of length $k$, that $X$ is not $k$-weakly defective, and that $\sigma_{k}(X)$ is a proper subvariety of $\mathbb{P} V$.

Then

$$
H^{0}\left(I_{Z^{\prime}} \otimes E\right) \otimes H^{0}\left(I_{Z^{\prime}} \otimes E^{*} \otimes L\right) \longrightarrow H^{0}\left(I_{Z^{\prime 2}} \otimes L\right)
$$


is surjective for general $Z^{\prime}$ of length $k^{\prime} \leq k$.

The analogous results hold in the symmetric and skew-symmetric cases.

Proof. It is enough to prove the case when $k^{\prime}=k-1$. Let $s \in H^{0}\left(I_{Z^{\prime}}^{2} \otimes L\right)$. By the assumption and Proposition 6.1.3 there are sections $s_{1}, \ldots, s_{t}$, with $t$ at most $\operatorname{dim} H^{0}\left(I_{Z^{\prime}}^{2} \otimes L\right)$, with singular points respectively $p_{1}, \ldots, p_{t}$ outside $Z^{\prime}$, such that $s=\sum_{i=1}^{t} s_{i}$. Let $Z_{i}=Z^{\prime} \cup\left\{p_{i}\right\}$ for $i=1, \ldots, t$. We may assume that the $p_{i}$ are in general linear position. By assumption $s_{i}$ is in the image of $H^{0}\left(I_{Z_{i}} \otimes E\right) \otimes H^{0}\left(I_{Z_{i}} \otimes E^{*} \otimes L\right) \longrightarrow H^{0}\left(I_{Z_{i}^{2}} \otimes L\right)$. So all $s_{i}$ come from $H^{0}\left(I_{Z^{\prime}} \otimes E\right) \otimes H^{0}\left(I_{Z^{\prime}} \otimes E^{*} \otimes L\right)$. The symmetric and skew-symmetric cases are analogous.

Example 6.2.2 (Examples where downward induction fails). Theorem 6.1.2 (ii) furnishes cases where the hypotheses of Lemma 6.2.1 are not satisfied. Let $k=9$ and $L=\mathcal{O}_{\mathbb{P}^{2}}(6)$. Here 9 general singular points impose independent conditions on sextics, but for $k^{\prime}=8$, all the sextics singular at eight general points have an additional singular point, namely the ninth point given by the intersection of all cubics through the eight points, so it is not general. Indeed, in this case $\sigma_{9}\left(v_{6}\left(\mathbb{P}^{2}\right)\right)$ is the catalecticant hypersurface of degree 10 given by the determinant of $\phi_{3,3} \in S^{3} \mathbb{C}^{3} \otimes S^{3} \mathbb{C}^{3}$, but the $9 \times 9$ minors of $\phi_{3,3}$ define a variety of dimension 24 which strictly contains $\sigma_{8}\left(v_{6}\left(\mathbb{P}^{2}\right)\right)$, which has dimension 23 .

Similarly, the $9 \times 9$ minors of $\phi_{2,2} \in S^{2} \mathbb{C}^{4} \otimes S^{2} \mathbb{C}^{4}$ define $\sigma_{8}\left(v_{4}\left(\mathbb{P}^{3}\right)\right)$, but the $8 \times 8$ minors of $\phi_{2,2}$ define a variety of dimension 28 which strictly contains $\sigma_{7}\left(v_{4}\left(\mathbb{P}^{3}\right)\right)$, which has dimension 27 .

\section{Proof of Theorem 1.2.3}

Recall $a=\left\lfloor\frac{n}{2}\right\rfloor, d=2 \delta+1, V=\mathbb{C}^{n+1}$. By Lemma 6.2.1, it is sufficient to prove the case $t=\left(\begin{array}{c}\delta+n \\ n\end{array}\right)$. Here $\left.E=\wedge^{n-a} Q(\delta)\right)$ where $Q \rightarrow \mathbb{P} V$ is the tautological quotient bundle.

By Theorem 5.4.3 it is sufficient to prove the map

$$
H^{0}\left(I_{Z} \otimes \wedge^{a} Q(\delta)\right) \otimes H^{0}\left(I_{Z} \otimes \wedge^{n-a} Q(\delta)\right) \rightarrow H^{0}\left(I_{Z}^{2}(2 \delta+1)\right)
$$

is surjective. In the case $n=2 a, a$ odd we have to prove that the map

$$
\wedge^{2} H^{0}\left(I_{Z} \otimes \wedge^{a} Q(\delta)\right) \rightarrow H^{0}\left(I_{Z}^{2}(2 \delta+1)\right)
$$

is surjective. The arguments are similar.

We need the following lemma, whose proof is given below:

Lemma 7.0.3. Let $Z$ be a set of $\left(\begin{array}{c}\delta+n \\ n\end{array}\right)$ points in $\mathbb{P}^{n}$ obtained as the intersection of $\delta+n$ general hyperplanes $H_{1}, \ldots, H_{\delta+n}(\delta \geq 1)$.

Let $h_{i} \in V^{*}$ be an equation for $H_{i}$. A basis of the space of polynomials of degree $2 \delta+1$ which are singular on $Z$ is given by $P_{I, J}:=\left(\prod_{i \in I} h_{i}\right) \cdot\left(\prod_{j \in J} h_{j}\right)$ where $I, J \subseteq\{1, \ldots \delta+n\}$ are multi-indices (without repetitions) satisfying $|I|=\delta+1,|J|=\delta$, and $|I \cap J| \leq \delta-1$ (that is $J$ cannot be contained in $I$ ).

We prove that the map (15) is surjective for $t=\left(\begin{array}{c}\delta+n \\ n\end{array}\right)$ by degeneration to the case when the $\left(\begin{array}{c}\delta+n \\ n\end{array}\right)$ points are the vertices of a configuration given by the union of $\delta+n$ general hyperplanes given by linear forms $h_{1}, \ldots, h_{\delta+n} \in V^{*}$.

First consider the case $n$ is even. For any multi-index $I \subset\{1, \ldots, \delta+n\}$ with $|I|=\delta$ write $q_{I}=\prod_{k \in I} h_{k} \in H^{0}(\mathcal{O}(\delta))$ and for any multi-index $H$, of length $a+1$, let $s_{H}$ be the section of $\wedge^{a} Q$ represented by the linear subspace of dimension $a-1$ given by $h_{H}=\left\{h_{i}=0, i \in H\right\}$. Indeed the linear subspace is a decomposable element of $\wedge^{a} V=H^{0}\left(\wedge^{a} Q\right)$. The section $s_{H}$ is represented by the Plücker coordinates of $h_{H}$.

The section $q_{I} s_{H} \in H^{0}\left(\wedge^{a} Q(\delta)\right)$ vanishes on the reducible variety consisting of the linear subspace $h_{H}$ of dimension $a-1$ and the degree $\delta$ hypersurface $q_{I}=0$. In particular, if $I \cap H=\emptyset$, then $q_{I} s_{H} \in H^{0}\left(I_{Z} \otimes \wedge^{a} Q(\delta)\right)$. 
Let $I=I_{0} \cup\{u\}$ with $\left|I_{0}\right|=\delta$, consider $|J|=\delta$ such that $u \notin J$ and $|I \cap J| \leq \delta-1$. It is possible to choose $K_{1}, K_{2}$ such that $\left|K_{1}\right|=\left|K_{2}\right|=a$ and such that $K_{1} \cap K_{2}=K_{1} \cap I=K_{2} \cap J=\emptyset$.

We get $q_{I_{0}} s_{\{u\} \cup K_{1}} \wedge q_{J} s_{\{u\} \cup K_{2}}$ is the degree $2 \delta+1$ hypersurface $q_{I_{0}} q_{J} h_{u}=q_{I} q_{J}$. By Lemma 7.0.3 these hypersurfaces generate $H^{0}\left(I_{Z}^{2}(2 \delta+1)\right)$. Hence $W_{Z}$ is surjective and the result is proved in the case $n$ is even.

When $n$ is odd, the morphism $A_{\phi}$ is represented by a rectangular matrix, and it induces a map $B: H^{0}\left(\wedge^{a} Q(\delta)\right) \otimes H^{0}\left(\wedge^{n-a} Q(\delta)\right) \rightarrow H^{0}(\mathcal{O}(2 \delta+1))$.

For any multi-index $H$, of length $n-a+1$, let $s_{H}$ be the section of $\wedge^{a} Q$ represented by the linear subspace of dimension $a-1$ given by $h_{H}=\left\{h_{i}=0, i \in H\right\}$. Indeed the linear subspace is a decomposable element of $\wedge^{a} V=H^{0}\left(\wedge^{a} Q\right)$. The section $s_{H}$ is represented by the Plücker coordinates of $h_{H}$.

The section $q_{I} s_{H} \in H^{0}\left(\wedge^{a} Q(\delta)\right)$ vanishes on the reducible variety consisting of the linear subspace $h_{H}$ of dimension $a-1$ and the degree $\delta$ hypersurface $q_{I}$. In particular, if $I \cap H=\emptyset$, then $q_{I} s_{H} \in H^{0}\left(I_{Z} \otimes \wedge^{a} Q(\delta)\right)$.

Let $I=I_{0} \cup\{u\}$ with $\left|I_{0}\right|=\delta$, consider $|J|=\delta$ such that $u \notin J$ and $|I \cap J| \leq \delta-1$. The modification to the above proof is that it is possible to choose $K_{1}, K_{2}$ such that $\left|K_{1}\right|=n-a=$ $a+1,\left|K_{2}\right|=a$ and such that $K_{1} \cap K_{2}=K_{1} \cap I=K_{2} \cap J=\emptyset$.

Then $q_{I_{0}} s_{\{u\} \cup K_{1}} \in H^{0}\left(\wedge^{a+1} Q(\delta)\right), q_{J} s_{\{u\} \cup K_{2}} \in H^{0}\left(\wedge^{a} Q(\delta)\right)$,

and $q_{I_{0}} s_{\{u\} \cup K_{1}} \wedge q_{J} s_{\{u\} \cup K_{2}}$ is the degree $2 \delta+1$ hypersurface $q_{I_{0}} q_{J} h_{u}=q_{I} q_{J}$. By Lemma 7.0.3 these hypersurfaces generate $H^{0}\left(I_{Z}^{2}(2 \delta+1)\right)$. Hence the map (15) is surjective and the result is proved.

Proof of Lemma 7.0.3. Let $Z$ be a set of $\left(\begin{array}{c}\delta+n \\ n\end{array}\right)$ points in $\mathbb{P}^{n}=\mathbb{P} V$ obtained as the intersection of $\delta+n$ general hyperplanes $H_{1}, \ldots, H_{\delta+n}(\delta \geq 1)$, and $h_{i} \in V^{*}$ is an equation for $H_{i}$.

We begin by discussing properties of the hypersurfaces through $Z$, which may be of independent interest.

\section{Proposition 7.0.4.}

(i) For $d \geq \delta$, the products $\prod_{i \in I} h_{i}$ for every $I \subseteq\{1, \ldots, n+\delta\}$ such that $|I|=d$ are independent in $S^{d} V^{*}$.

(ii) For $d \leq \delta$, the products $\prod_{i \in I} h_{i}$ for every $I \subseteq\{1, \ldots, n+\delta\}$ such that $|I|=d$ span $S^{d} V^{*}$.

Proof. Write $h_{I}=\prod_{\in I} h_{i}$. Consider a linear combination $\sum_{I} a_{I} h_{I}=0$. Let $d \geq \delta$. If $d \geq n+\delta$ the statement is vacuous, so assume $d<n+\delta$. For each $I_{0}$ such that $\left|I_{0}\right|=d$ there is a point $P_{0} \in V$ such that $h_{i}\left(P_{0}\right)=0$ for $i \notin I_{0}$ and $h_{i}\left(P_{0}\right) \neq 0$ for $i \in I_{0}$. The equality $\sum_{I} a_{I} h_{I}\left(P_{0}\right)=0$ implies $a_{I_{0}}=0$, proving (i). For $d=\delta$, (ii) follows as well by counting dimensions, as $\operatorname{dim} S^{\delta} V^{*}=\left(\begin{array}{c}\delta+n \\ n\end{array}\right)$. For $d \leq \delta-1$ pick $f \in S^{d} V^{*}$. By the case already proved, there exist coefficients $a_{I}, b_{J}$ such that

$$
f \prod_{i=1}^{\delta-d} h_{i}=\sum_{I} a_{I} h_{I}+\sum_{J} b_{J} h_{J}
$$

where the first sum is over all $I$ with $|I|=\delta$ such that $\{1, \ldots, \delta-d\} \subseteq I$ and the second sum over all $J$ with $|J|=\delta$ such that $\{1, \ldots, \delta-d\} \nsubseteq J$.

For every $J_{0}$ such that $\left|J_{0}\right|=\delta$ and $\{1, \ldots, \delta-d\} \nsubseteq J_{0}$ there is a (unique) point $\left[P_{0}\right]$ such that $h_{i}\left(P_{0}\right)=0$ for $i \notin J_{0}$ and $h_{i}\left(P_{0}\right) \neq 0$ for $i \in J_{0}$. In particular $\prod_{i=1}^{\delta-d} h_{i}\left(P_{0}\right)=0$ and substituting $P_{0}$ into (17) shows $b_{J_{0}}=0$, for each $J_{0}$. Thus

$$
f \prod_{i=1}^{\delta-d} h_{i}=\sum_{I} a_{I} h_{I}
$$


and dividing both sides by $\prod_{i=1}^{\delta-d} h_{i}$ proves (ii).

Recall that the subspace of $S^{d} V^{*}$ of polynomials which pass through $p$ points and are singular through $q$ points has codimension $\leq \min \left(\left(\begin{array}{c}n+d \\ n\end{array}\right), p+q(n+1)\right)$. When equality holds one says that the conditions imposed by the points are independent and that the subspace has the expected codimension.

Let $I_{Z}$ denote the ideal sheaf of $Z$ and $I_{Z}(d)=I_{Z} \otimes \mathcal{O}_{\mathbb{P}^{n}}(d)$. These sheaves have cohomology groups $H^{p}\left(I_{Z}(d)\right)$, where in particular $H^{0}\left(I_{Z}(d)\right)=\left\{P \in S^{d} V^{*} \mid Z \subseteq \operatorname{Zeros}(P)\right\}$.

Proposition 7.0.5. Let $Z$ be a set of $\left(\begin{array}{c}n+\delta \\ n\end{array}\right)$ points in $\mathbb{P}^{n}$ obtained as above. Then

(i) $H^{0}\left(I_{Z}(d)\right)=0$ if $d \leq \delta$.

(ii) $H^{0}\left(I_{Z}(\delta+1)\right)$ has dimension $\left(\begin{array}{c}\delta+n \\ \delta+1\end{array}\right)$ and it is generated by the products $h_{I}$ with $|I|=\delta+1$.

(iii) If $d \geq \delta+1$ then $H^{0}\left(I_{Z}(d)\right)$ is generated in degree $\delta+1$, that is the natural morphism $H^{0}\left(I_{Z}(\delta+1)\right) \otimes H^{0}\left(\mathcal{O}_{\mathbb{P}^{n}}(d-\delta-1)\right) \longrightarrow H^{0}\left(I_{Z}(d)\right)$ is surjective.

Proof. Consider the exact sequence of sheaves

$$
0 \longrightarrow I_{Z}(d) \longrightarrow \mathcal{O}_{\mathbb{P}^{n}}(d) \longrightarrow \mathcal{O}_{Z}(d) \longrightarrow 0 .
$$

Since $Z$ is finite, $\operatorname{dim} H^{0}\left(\mathcal{O}_{Z}(d)\right)=\left(\begin{array}{c}\delta+n \\ n\end{array}\right)=\operatorname{deg} Z$ for every $d$.

The space $H^{0}\left(\mathcal{O}_{\mathbb{P}^{n}}(\delta)\right)$ has dimension $\left(\begin{array}{c}\delta+n \\ n\end{array}\right)$ and by Proposition 7.0.4, for every $I$ with $|I|=\delta$ the element $h_{I}$ vanishes on all the points of $Z$ with just one exception, given by $\cap_{j \notin I} H_{j}$. Hence the restriction map $H^{0}\left(\mathcal{O}_{\mathbb{P}^{n}}(\delta)\right) \longrightarrow H^{0}\left(\mathcal{O}_{Z}(\delta)\right)$ is an isomorphism. It follows that $H^{0}\left(I_{Z}(\delta)\right)=$ $H^{1}\left(I_{Z}(\delta)\right)=0$, and thus $H^{0}\left(I_{Z}(d)\right)=0$ for $d<\delta$ proving (i).

Since $\operatorname{dim} Z=0, H^{i}\left(\mathcal{O}_{Z}(k)\right)=0$ for $i \geq 1$, and all $k$. ¿From this it follows that $H^{i}\left(I_{Z}(\delta-\right.$ $i+1))=0$ for $i \geq 2$, because $H^{i}\left(\mathcal{O}_{\mathbb{P}^{n}}(\delta-i+1)\right)=0$. The vanishing for $i=1$ was proved above, so that $I_{Z}$ is $(\delta+1)$-regular and by the Castelnuovo-Mumford criterion [37, Chap. 14] $I_{Z}(\delta+1)$ is globally generated, $H^{1}\left(I_{Z}(k)\right)=0$ for $k \geq \delta+1$, and part (iii) follows.

In order to prove (ii), consider the products $h_{I}$ with $|I|=\delta+1$, which are independent by Proposition 7.0.4.i, so they span a $\left(\begin{array}{c}\delta+n \\ \delta+1\end{array}\right)$-dimensional subspace of $H^{0}\left(I_{Z}(\delta+1)\right)$.

The long exact sequence in cohomology implies

$$
\begin{aligned}
h^{0}\left(I_{Z}(\delta+1)\right) & =h^{0}\left(\mathcal{O}_{\mathbb{P}^{n}}(\delta+1)\right)-h^{0}\left(\mathcal{O}_{Z}(\delta+1)\right)+h^{1}\left(I_{Z}(\delta+1)\right) \\
& =\left(\begin{array}{c}
\delta+1+n \\
n
\end{array}\right)-\left(\begin{array}{c}
\delta+n \\
n
\end{array}\right)+0=\left(\begin{array}{c}
\delta+n \\
\delta+1
\end{array}\right)
\end{aligned}
$$

which concludes the proof.

Proposition 7.0.6. Notations as above. Let $L_{0} \subset\{1, \ldots, n+\delta\}$ have cardinality $n$.

The space of polynomials of degree $\delta+1$ which pass through the points $y_{L}$ and are singular at $y_{L_{0}}$ has a basis given by the products $\prod_{i \in J} h_{i}$ for every $J \subseteq\{1, \ldots, n+\delta\}$ such that $|J|=\delta+1$ and $\#\left(J \cap L_{0}\right) \geq 2$. This space has the expected codimension $\left(\begin{array}{c}\delta+n \\ n\end{array}\right)+n$.

Proof. Note that if $|J|=\delta+1$ then $\#\left(J \cap L_{0}\right) \geq 1$ and equality holds just for the $n$ products $h_{i} h_{L_{0}^{c}}$ for $i \in L_{0}$, where $L_{0}^{c}=\{1, \ldots, n+\delta\} \backslash L_{0}$. Every linear combination of the $h_{J}$ which has a nonzero coefficient in these $n$ products is nonsingular at $\left[y_{L_{0}}\right]$.

Hence the products which are different from these $n$ generate the space of polynomials of degree $\delta+1$ which pass through the points $\left[y_{L}\right]$ and are singular at $\left[y_{L_{0}}\right]$.

There are $\left(\begin{array}{c}\delta+n \\ n-1\end{array}\right)-n$ such polynomials, which is the expected number $\left(\begin{array}{c}\delta+n+1 \\ n\end{array}\right)-(n+1)-$ $\left[\left(\begin{array}{c}\delta+n \\ n\end{array}\right)-1\right]$. Since the codimension is always at most the expected one, it follows that these generators give a basis. 
In the remainder of this section, we use products $h_{I}$ where $I$ is a multi-index where repetitions are allowed. Given such a multi-index $I=\left\{i_{1}, \ldots, i_{|I|}\right\}$, we write $h_{I}=h_{1}^{k_{1}} \cdots h_{n+\delta}^{k_{n+\delta}}$, where $j$ appears $k_{j}$ times in $I$ and we write $|I|=k_{1}+\cdots+k_{n+\delta}$. The support $s(I)$ of a multi-index $I$ is the set of $j \subset\{1, \ldots, n+\delta\}$ with $k_{j}>0$.

An immediate consequence of (ii) and (iii) of Proposition 7.0.5 is:

Corollary 7.0.7. For $d \geq \delta+1$, the vector space $H^{0}\left(I_{Z}(d)\right)$ is generated by the monomials $h_{I}$ with $|I|=d$ and $|s(I)| \geq \delta+1$.

Proposition 7.0.8. The space of polynomials of degree $2 \delta+1$ which contain $Z$ is generated by products $h_{I}$ with $|I|=2 \delta+1,|s(I)| \geq \delta+1$, and all the exponents in $h_{I}$ are at most 2 .

Proof. By Corollary 7.0.7, it just remains to prove the statement about the exponents. Let $h_{I}=\prod_{i=1}^{\delta+n} h_{i}^{k_{i}}$ and let $n_{j}(I)=\#\left\{i \mid k_{i}=j\right\}$ for $j=0, \ldots, 2 \delta+1$. Hence $\sum_{j \geq 1} j n_{j}(I)=2 \delta+1$ and $\sum_{j>1} n_{j}(I) \geq \delta+1$.

Assume that $\gamma:=\sum_{j \geq 3} n_{j}(I)>0$. It is enough to show that $h_{I}=\sum c_{J} h_{J}$ where every $J$ which appears in the sum satisfies $|s(J)| \geq \delta+1,|J|=2 \delta+1$ and $\sum_{j \geq 3} n_{j}(J)<\gamma$.

Indeed

$2 \delta+1=n_{1}(I)+2 n_{2}(I)+3 n_{3}(I)+\ldots \geq n_{1}(I)+2 n_{2}(I)+3 \gamma \geq\left(\delta+1-n_{2}(I)-\gamma\right)+2 n_{2}(I)+3 \gamma$

that is, $n_{2}(I)+\gamma \leq \delta-\gamma \leq \delta-1$. Hence there at least $n+1$ forms $h_{i}$ which appear with exponent at most one in $h_{I}$. We can express all the remaining forms $h_{s}$ as linear combinations of these $n+1$ forms. By expressing a form with exponent at least 3 as linear combination of these $n+1$ linear forms $h_{i}$, we get $h_{I}=\sum c_{J} h_{J}$ where each summand has the required properties.

To better understand the numbers in the following proposition, recall that $\sum_{i=1}^{\delta+1}\left(\begin{array}{c}\delta+n-i \\ n-1\end{array}\right)=$ $\left(\begin{array}{c}\delta+n \\ n\end{array}\right)$ as taking $E=\mathbb{C}^{n}, F=\mathbb{C}^{1}$, one has $S^{n}(E+F)=S^{n} E \oplus S^{n-1} E \otimes F \oplus S^{n-2} E \otimes S^{2} F \oplus \cdots \oplus$ $S^{n} F$.

Proposition 7.0.9. For $k=0, \ldots, \delta$, let $I_{\delta, k, n}$ be the linear system of hypersurfaces of degree $2 \delta+1-k$ which contain $Z$ and are singular on the points of $Z$ which lie outside $\cup_{i=1}^{k} H_{i}$. The space $I_{\delta, k, n}$ has the expected codimension $(n+1)\left(\begin{array}{c}\delta+n \\ n\end{array}\right)-n \sum_{i=1}^{k}\left(\begin{array}{c}\delta+n-i \\ n-1\end{array}\right)$.

Proof. For $k=\delta$ the assertion is Proposition 7.0.6 with $L_{0}=\{\delta+1, \ldots, \delta+n\}$. We work by induction on $n$ and by descending induction on $k$ (for fixed $\delta$ ). We restrict to the last hyperplane $H_{\delta+n}$.

Let $V_{\delta, k+1, n} \supseteq I_{\delta, k+1, n}$ denote the set of hypersurfaces of degree $2 \delta-k$ which pass through $Z \backslash\left\{H_{\delta+n} \cap\left(\cup_{i=1}^{k} H_{i}\right)\right\}$ and are singular on the points of $Z$ which lie outside $\left(\cup_{i=1}^{k} H_{i}\right) \cup\left\{H_{\delta+n}\right\}$. We have the exact sequence

$$
0 \longrightarrow V_{\delta, k+1, n} \stackrel{\psi}{\longrightarrow} I_{\delta, k, n} \stackrel{\phi}{\longrightarrow} I_{\delta, k, n-1}
$$

where $\phi$ is the restriction to $\mathbb{C}^{n} \subset \mathbb{C}^{n+1}$, and $\psi$ is the multiplication by $h_{\delta+n}$.

Since by induction $I_{\delta, k+1, n}$ has the expected codimension in $S^{2 \delta-k} V^{*}$ it follows that also $V_{\delta, k+1, n}$ has the expected codimension

$$
n\left[\left(\begin{array}{c}
\delta+n-1 \\
n
\end{array}\right)-\sum_{i=1}^{k}\left(\begin{array}{c}
\delta+n-1-i \\
n-1
\end{array}\right)\right]+\left(\begin{array}{c}
\delta+n \\
n
\end{array}\right)-\sum_{i=1}^{k}\left(\begin{array}{c}
\delta+n-1-i \\
n-2
\end{array}\right)
$$

because the conditions imposed by $V_{\delta, k+1, n}$ are a subset of the conditions imposed by $I_{\delta, k+1, n}$ and a subset of a set of independent conditions still consists of independent conditions.

Since the codimension of $I_{\delta, k, n-1}$ in $S^{2 \delta+1-k} \mathbb{C}^{n}$ is the expected one, it follows that the codimension of $I_{\delta, k, n}$ in $S^{2 \delta+1-k} V^{*}$ is at least the expected one, hence equality holds. 
The following Corollary proves Lemma 7.0.3.

Corollary 7.0.10. Let $Z$ be a set of $\left(\begin{array}{c}\delta+n \\ n\end{array}\right)$ points in $\mathbb{P}^{n}=\mathbb{P} V$ obtained as the intersection of $\delta+n$ general hyperplanes $H_{1}, \ldots, H_{\delta+n}(\delta \geq 1)$, and let $h_{i} \in V^{*}$ be an equation of $H_{i}$. The linear system of hypersurfaces of degree $2 \delta+1$ which are singular on $Z$ has the expected codimension $(n+1)\left(\begin{array}{c}\delta+n \\ n\end{array}\right)$ and it is generated by the products $h_{I}=\prod_{i \in I} h_{i}$ with $I$ a multi-index allowing repetitions such that $|I|=2 \delta+1$ and the support of $I$ is at least $\delta+2$ and all the exponents are at most two.

Proof. The statement about the codimension is the case $k=0$ of Proposition 7.0.9. Note that every product $h_{I}$ with $I$ such that $|I|=2 \delta+1$ and $s(I) \geq \delta+2$ is singular at any $P \in Z$ because $h_{I}$ contains at least two factors which vanish at $P$. Let $f$ be a homogeneous polynomial of degree $2 \delta+1$ which is singular on $Z$. By Proposition 7.0.8 we have the decomposition $f=\sum_{|s(I)| \geq \delta+2} a_{I} h_{I}+\sum_{|s(I)|=\delta+1} b_{I} h_{I}$, where all the exponents are at most 2 . Let $I_{0}$ be in the second summation with $\left|s\left(I_{0}\right)\right|=\delta+1$. There is a unique $h_{i}$ appearing in $h_{I_{0}}$ with exponent 1 and $n-1$ hyperplanes appearing with exponent 0 . Let $P_{0}$ be the point where these $1+(n-1)=n$ hyperplanes meet. It follows that $h_{I_{0}}$ is nonsingular at $P_{0}$ while, for all the other summands, $h_{I}$ is singular in $P_{0}$. It follows that $b_{I_{0}}=0$ and $f=\sum_{|s(I)| \geq \delta+2} a_{I} h_{I}$ as we wanted.

\section{Construction of $A_{v}^{E}$ via a presentation of $E$}

8.1. Motivating example. Set $\operatorname{dim} V=n+1, d=2 \delta+1$ and $a=\left\lfloor\frac{n}{2}\right\rfloor$. We saw in $\S 4$ that the inclusion $S^{d} V \subset S_{\delta+1,1^{n-a}} V^{*} \otimes S_{\delta+1,1^{a}} V$ yields new modules of equations for $\sigma_{r}\left(v_{d}(\mathbb{P} V)\right)$. The relevant vector bundle here is $E=E_{\delta \omega_{1}+\omega_{n-a+1}}=\wedge^{a} Q(\delta)$ where $Q$ is the tautological quotient bundle. The equations were initially presented without reference to representation theory using (2). Note that $\operatorname{rank}\left(Y F_{d, n}\left(w^{d}\right)\right)=\left(\begin{array}{l}n \\ a\end{array}\right)=\operatorname{rank}(E)$.

This map between larger, but more elementary, spaces has the same rank properties as the map $S_{\delta+1,1^{n-a}} V^{*} \rightarrow S_{\delta+1,1^{a}} V$, because when one decomposes the spaces in this map as $S L(V)$ modules, the other module maps are zero.

Observe that

$$
\begin{aligned}
S^{\delta} V^{*} \otimes \wedge^{a} V & =H^{0}\left(\mathcal{O}(\delta)_{\mathbb{P} V} \otimes \wedge^{a} V\right), \\
\text { and }\left(S^{\delta} V \otimes \wedge^{a+1} V\right)^{*} & =H^{0}\left(\mathcal{O}(\delta)_{\mathbb{P} V} \otimes \wedge^{n-a+1} V\right) .
\end{aligned}
$$

The vector bundle $E=\wedge^{a} Q(\delta)$ may be recovered as the image of the map

$$
L_{1}=\mathcal{O}(\delta) \otimes \wedge^{a} V \stackrel{p_{E}}{\longrightarrow} \mathcal{O}(\delta+1) \otimes \wedge^{a+1} V=L_{0} .
$$

8.2. The general case. In the general set-up, in order to factorize $E$, one begins with a base line bundle $M \rightarrow X$, which is $\mathcal{O}_{\mathbb{P} W}(1)$ when $X=v_{d}(\mathbb{P} W) \subset \mathbb{P} S^{d} W=\mathbb{P} V$, and more generally is the ample generator of the Picard group if $X$ has Picard number one (e.g., rational homogeneous varieties $G / P$ with $P$ maximal).

The key is to realize $E$ as the image of a map

$$
p_{E}: L_{1} \rightarrow L_{0}
$$

where the $L_{i}$ are direct sums of powers of $M$ and

$$
\operatorname{Image}\left(p_{E}\right)=E \text {. }
$$

By construction, for $x \in \hat{X}, \operatorname{rank}\left(p_{E}(x)\right)=e$. Let $v \in V=H^{0}(X, L)^{*}$. Compose the map $H^{0}\left(L_{0}\right) \otimes H^{0}(L)^{*} \rightarrow H^{0}\left(L_{0}^{*} \otimes L\right)^{*}$ with the map on sections induced from $p_{E}$, to obtain

$$
\begin{gathered}
P_{v}^{E}: H^{0}\left(L_{1}\right) \rightarrow H^{0}\left(L_{0}^{*} \otimes L\right)^{*} \\
\text { and observe the linearity } P_{s v_{1}+t v_{2}}^{E}=s P_{v_{1}}^{E}+t P_{v_{2}}^{E} \text { for } v_{1}, v_{2} \in V, s, t \in \mathbb{C} \text {. }
\end{gathered}
$$


8.3. Case $X=v_{d}(\mathbb{P} W)$. In the case $(X, M)=(\mathbb{P} W, \mathcal{O}(1))$, and $L=\mathcal{O}(d)$, this means

$$
L_{j}=\oplus(\mathcal{O}(i))^{\oplus a_{j}^{i}}
$$

for some finite set of natural numbers $a_{1}^{i}, a_{2}^{i}$. For the examples in this paper there is just one term in the summand, e.g., in $\S 8.1, L_{1}=\mathcal{O}(\delta)^{\oplus\left(\begin{array}{c}n+1 \\ a\end{array}\right)}, L_{0}=\mathcal{O}(\delta+1)^{\oplus\left(\begin{array}{c}n+1 \\ a+1\end{array}\right)}$.

When $X=v_{d}(\mathbb{P} W), P_{v}^{E}$ may be written explicitly in bases as follows:

Let $L_{1}=\oplus_{j=1}^{m_{1}} \mathcal{O}\left(b_{j}\right)$ and $L_{0}=\oplus_{i=1}^{m_{0}} \mathcal{O}\left(a_{i}\right)$. Let $p_{i j} \in S^{a_{i}-b_{j}} W^{*}$ denote the map $\mathcal{O}\left(b_{j}\right) \rightarrow$ $\mathcal{O}\left(a_{i}\right)$, given by the composition

$$
\mathcal{O}\left(b_{j}\right) \rightarrow L_{1} \stackrel{p_{E}}{\longrightarrow} L_{0} \rightarrow \mathcal{O}\left(a_{i}\right)
$$

Then, for any $\phi \in S^{d} W, P_{v}^{E}(\phi)$ is obtained by taking a matrix of $m_{1} \times m_{0}$ blocks with the $(i, j)$-th block a matrix representing the catalecticant $\phi_{b_{j}, d-a_{i}} \in S^{b_{j}} W^{*} \otimes S^{d-a_{i}} W^{*} \otimes S^{d} W$ contracted by $p_{i j}$ so the new matrix is of size $h^{0}\left(L_{0}^{*} \otimes L\right) \times h^{0}\left(L_{1}\right)$ with scalar entries. See Examples 1.2.1 and 8.4.4 for explicit examples.

8.4. Rank and irreducible component theorems in the presentation setting. Under mild assumptions, the following proposition shows that $P_{v}^{E}$ can be used in place of $A_{v}^{E}$ in the theorems of $\S 5$.

Proposition 8.4.1. Notations as above. Assume that $H^{0}\left(L_{1}\right) \stackrel{i}{\longrightarrow} H^{0}(E)$ and $H^{0}\left(L_{0}^{*} \otimes L\right) \stackrel{j}{\longrightarrow} H^{0}\left(E^{*} \otimes\right.$ L) are surjective.

Then the rank of $A_{v}^{E}$ equals the rank of $P_{v}^{E}$, so that the size $(k e+1)$-minors of $P_{v}^{E}$ give equations for $\sigma_{k}(X)$.

If $(E, L)$ is a symmetric (resp. skew-symmetric) pair then there exists a symmetric (resp. skew-symmetric) presentation where $L_{1} \simeq L_{0}^{*} \otimes L$ and $P_{v}^{E}$ is symmetric (resp. skew-symmetric).

Proof. Consider the commutative diagram

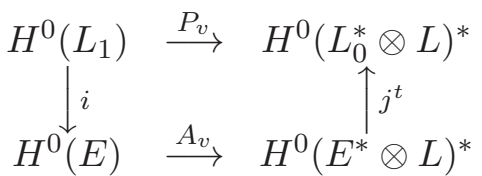

The assumptions $i$ is surjective and $j^{t}$ is injective $\operatorname{imply} \operatorname{rank}\left(A_{v}\right)=\operatorname{rank}\left(P_{v}\right)$. The symmetric and skew assertions are clear.

With the assumptions of Proposition 8.4.1, the natural map

$$
H^{0}\left(L_{1}\right) \otimes H^{0}\left(L_{0}^{*}\right) \rightarrow H^{0}(E) \otimes H^{0}\left(E^{*} \otimes L\right)
$$

is surjective and the affine conormal space at $v$ is the image of $\operatorname{ker} P_{v} \otimes\left(\operatorname{Im} P_{v}\right)^{\perp} \rightarrow H^{0}(L)$.

The following variant of Proposition 5.1.1 is often easy to implement in practice.

Theorem 8.4.2. Notations as above. Let $v \in \sigma_{k}(X)$. Assume the maps $H^{0}\left(L_{1}\right) \longrightarrow H^{0}(E)$ and $H^{0}\left(L_{0}^{*} \otimes L\right) \longrightarrow H^{0}\left(E^{*} \otimes L\right)$ are surjective.

(i) If the rank of ker $P_{v} \otimes\left(\operatorname{Im} P_{v}\right)^{\perp} \stackrel{g}{\longrightarrow} H^{0}(L)$ at $v$ equals the codimension of $\sigma_{k}(X)$, then $\sigma_{k}(X)$ is an irreducible component of $\operatorname{Rank}_{k}(E)$ passing through $v$.

(ii) If $(E, L)$ is a symmetric (resp. skew-symmetric) pair, assume that the rank of $S^{2}$ (ker $\left.P_{v}\right) \rightarrow$ $H^{0}(L)$ (resp. of $\left.\wedge^{2}\left(\operatorname{ker} P_{v}\right) \rightarrow H^{0}(L)\right)$ at $v$ coincides with the codimension of $\sigma_{k}(X)$.

Then $\sigma_{k}(X)$ is an irreducible component of $\operatorname{Rank}_{k}(E)$ passing through $v$.

Note that in the skew-symmetric case, $\operatorname{Rank}_{k}(E)$ is defined by sub-Pfaffians. 
Remark 8.4.3. In Theorem 8.4.2, the rank of the maps is always bounded above by the codimension of $\sigma_{k}(X)$, as the rank of the maps is equal to the dimension of the conormal space of $\operatorname{Rank}_{k}(E)$ at $v$.

The previous theorem can be implemented in concrete examples. Indeed the pairing $g$, appearing in the theorem, can be described as follows: given $f \in \operatorname{ker} P_{v} \subset H^{0}\left(L_{1}\right), h \in\left(\operatorname{Im} P_{v}\right)^{\perp} \subset$ $H^{0}\left(L_{0}^{*} \otimes L\right)$, and $\phi \in H^{0}(L)^{*}$, one has $g(f, h)(\phi)=h\left[P_{\phi}(f)\right]$.

Example 8.4.4. [Cubic 3-folds revisited] Let $\operatorname{dim} V=5$, let $E=\Omega^{2}(4)=\wedge^{2} T^{*} \mathbb{P} V(4)=E_{\omega_{2}}$ on $X=\mathbb{P}^{4}$, the pair $(E, \mathcal{O}(3))$ is a symmetric pair presented by

$$
L_{1}=\mathcal{O}(1) \otimes \wedge^{3} V \stackrel{p}{\longrightarrow} \mathcal{O}(2) \otimes \wedge^{2} V=L_{0} .
$$

Choose a basis $x_{0}, \ldots, x_{4}$ of $V$, so $p$ is represented by the $10 \times 10$ symmetric matrix called $K_{4}$ in the introduction. Let $L=\mathcal{O}(3)$, for any $\phi \in S^{3} V^{*}$ the map $A_{\phi}^{E}$ is the skew-symmetric morphism from $H^{0}\left(\Omega^{2}(4)\right)$ to its dual $H^{0}\left(\Omega^{3}(5)\right)^{*}$ where both have dimension 45 and $P_{\phi}$ is represented by the $50 \times 50$ block matrix where the entries $\pm x_{i}$ in $K_{4}$ are replaced with $\pm\left(\frac{\partial \phi}{\partial x_{j}}\right)_{1,1}$, which are $5 \times 5$ symmetric catalecticant matrices of the quadric $\frac{\partial \phi}{\partial x_{j}}$. In [40] it is shown that a matrix representing $A_{\phi}^{E}$ is obtained from the matrix representing $P_{\phi}$ by deleting five suitably chosen rows and columns. Here $\operatorname{det}\left(A_{\phi}\right)$ is the cube of the degree 15 equation of $\sigma_{7}\left(v_{3}\left(\mathbb{P}^{4}\right)\right)$.

Note that when $\phi=x_{0}^{3}$ is a cube, then the rank of $A_{\phi}^{E}$ and the rank of $P_{\phi}$ are both equal to 6 , which is the rank of $\Omega^{2}(3)$.

\section{Decomposition of POlynomials into A SUM OF POWERS}

Having a presentation for $E$ enables one to reduce the problem of decomposing a polynomial into a sum of powers to a problem of solving a system of polynomial equations (sometimes linear) as we explain in this section. We begin with a classical example:

9.1. Catalecticant. Let $\phi \in S^{d} W$ be a general element of $\hat{\sigma}_{k}\left(v_{d}\left(\mathbb{P}^{n}\right)\right)$ so it can be written as $\phi=\sum_{i=1}^{k} l_{i}^{d}$. Let $Z=\left\{l_{1}, \ldots, l_{k}\right\}$, let $\delta=\left\lfloor\frac{d}{2}\right\rfloor$, and let $k \leq\left(\begin{array}{c}\delta+n-1 \\ n\end{array}\right)$. Take $E=\mathcal{O}(\delta)$ so $A_{\phi}^{E}: H^{0}(\mathcal{O}(\delta)) \rightarrow H^{0}(\mathcal{O}(d-\delta))^{*}$. Then ker $A_{\phi}=H^{0}\left(I_{Z}(\delta)\right)$ and ker $A_{\phi} \cap v_{\delta}(\mathbb{P} W)=\left\{l_{1}^{\delta}, \ldots, l_{k}^{\delta}\right\}$. Thus if one can compute the intersection, one can recover $Z$ and the decomposition. For a further discussion of these ideas see [38].

9.2. $Y F_{d, n}$. For $\phi \in S^{d} W$, let $\delta=\left\lfloor\frac{d-1}{2}\right\rfloor, a=\left\lfloor\frac{n}{2}\right\rfloor, k \leq\left(\begin{array}{c}\delta+n \\ n\end{array}\right)$ and take $E=\wedge^{a} Q(\delta)$, so $A_{\phi}: H^{0}\left(\wedge^{a} Q(\delta)\right) \rightarrow H^{0}\left(\wedge^{n-a} Q(d-\delta)\right)$, then ker $A_{\phi}=H^{0}\left(I_{Z} \otimes \wedge^{a} Q(\delta)\right)$ so that $Z$ can be recovered as the base locus of $\operatorname{ker} A_{\phi}$.

9.3. General quintics in $S^{5} \mathbb{C}^{3}$. A general element $\phi \in S^{5} \mathbb{C}^{3}$ is a unique sum of seven fifth powers (realized by Hilbert, Richmond and Palatini $[24,44,42]$ ), write $\phi=x_{1}^{5}+\cdots+x_{7}^{5}$. Here is an algorithm to compute the seven summands:

Let $E=Q(2)$ and consider the skew-symmetric morphism $A_{\phi}^{E}: H^{0}(Q(2)) \rightarrow H^{0}(Q(2))^{*}$. (Note that $H^{0}(Q(2))=S_{3,2} V$ as an $S L_{3}$-module, and that has dimension 15.) For a general $\phi$ the kernel of $A_{\phi}$ has dimension one and we let $s \in H^{0}(Q(2))$ denote a section spanning it. By Proposition 5.4.1, the seven points where s vanishes correspond to the seven summands. Explicitly, $s$ corresponds to a morphism $f: S^{2} W \rightarrow W$ such that the set $\left\{v \in W \mid f\left(v^{2}\right) \in<v>\right\}$ consists of the seven points $P_{1}, \ldots, P_{7}$. 
One way to describe the seven points is to consider the map

$$
\begin{aligned}
F_{\phi}: \mathbb{P} W & \rightarrow \mathbb{P}\left(\mathbb{C}^{2} \otimes W\right) \\
{[v] } & \mapsto\left[v \otimes e_{1}+f\left(v^{2}\right) \otimes e_{2}\right]
\end{aligned}
$$

where $\mathbb{C}^{2}$ has basis $e_{1}, e_{2}$. Then, the seven points are the intersection $F_{\phi}(\mathbb{P} W) \cap S e g\left(\mathbb{P}^{1} \times \mathbb{P} W\right)$, which is the set of two by two minors of a matrix with linear and quadratic entries. Giving $W^{*}$ the basis $x_{0}, x_{1}, x_{2}$, the matrix is of the form

$$
\left[\begin{array}{lll}
x_{0} & x_{1} & x_{2} \\
q_{0} & q_{1} & q_{2}
\end{array}\right]
$$

where the $q_{j}$ are of degree two. In practice this system is easily solved with, e.g. Maple.

\section{Grassmannians}

10.1. Skew-flattenings. Some natural equations for $\sigma_{r}(G(k, W)) \subset \mathbb{P} \wedge^{k} W$ are obtained from the inclusions $\wedge^{k} W \subset \wedge^{a} W \otimes \wedge^{k-a} W$ which we will call a skew-flattening. Let $W=\mathbb{C}^{n+1}$, let $\omega_{i}$, $1 \leq i \leq n$ denote the fundamental weights of $\mathfrak{s l}(W)$, let $E_{\lambda}$ denote the irreducible vector bundle induced from the $P$-module of highest weight $\lambda$. The skew-flattenings correspond to the bundle $E_{\omega_{a}} \rightarrow G(k, W)$. Note that $\operatorname{rank}\left(E_{\omega_{a}}\right)=\left(\begin{array}{l}k \\ a\end{array}\right)$, as $E_{\omega_{a}}$ is isomorphic to the $a$-th exterior power of the dual of the universal sub-bundle of rank $k$. The map $v_{a, k-a}=A_{v}^{E}: \wedge^{a} W^{*} \rightarrow \wedge^{k-a} W$ thus has rank $\left(\begin{array}{l}k \\ a\end{array}\right)$ (this is also easy to see directly). Since $\operatorname{dim} \wedge^{a} W=\left(\begin{array}{c}n+1 \\ k\end{array}\right)$, one obtains equations possibly up to the $\left(\begin{array}{c}n+1 \\ k\end{array}\right) /\left(\begin{array}{l}k \\ a\end{array}\right)$-th secant variety.

The case $k=2$ is well known, $I\left(\sigma_{r}(G(2, W))\right)$ is generated in degree $r+1$ by sub-Pfaffians of size $2 r+2$.

Let $E \in G(k, W)=\mathbb{G}\left(\mathbb{P}^{k-1}, \mathbb{P} W\right)$. We slightly abuse notation and also write $E \subset W$ for the corresponding linear subspace. Then

$$
\hat{N}_{E}^{*} G(k, W)=\wedge^{2}\left(E^{\perp}\right) \wedge\left(\wedge^{k-2} W^{*}\right) \subset \wedge^{k} W^{*} .
$$

Now let $\left[E_{1}+E_{2}\right] \in \sigma_{2}(G(k, W))$. By Terracini's lemma $\hat{N}_{\left[E_{1}+E_{2}\right]}^{*} G(k, W)=\hat{N}_{E_{1}}^{*} G(k, W) \cap$ $\hat{N}_{E_{2}}^{*} G(k, W)$. Let $U_{12}=E_{1}{ }^{\perp} \cap E_{2}{ }^{\perp}$, and $U_{j}=E_{j}{ }^{\perp}$. We have

$\hat{N}_{\left[E_{1}+E_{2}\right]}^{*} G(k, W)=\left(\wedge^{2} U_{12}\right) \wedge\left(\wedge^{k-2} W^{*}\right)+U_{12} \wedge U_{1} \wedge U_{2} \wedge\left(\wedge^{k-3} W^{*}\right)+\left(\wedge^{2} U_{1}\right) \wedge\left(\wedge^{2} U_{2}\right) \wedge\left(\wedge^{k-4} W^{*}\right)$.

To see this, note that each term must contain a $\wedge^{2}\left(E_{1}^{\perp}\right)$ and a $\wedge^{2}\left(E_{2}{ }^{\perp}\right)$. The notation is designed so that $\wedge^{2}\left(E_{j}{ }^{\perp}\right)$ will appear if the $j$ index occurs at least twice in the $U$ 's. Similarly below, one takes all combinations of $U$ 's such that each of 1,2,3 appears twice in the expression.

With similar notation,

$$
\begin{aligned}
N_{\left[E_{1}+E_{2}+E_{3}\right]}^{*} G(k, W)= & \left(\wedge^{2} U_{123}\right) \wedge\left(\wedge^{k-2} W^{*}\right) \\
& +\left[U_{123} \wedge U_{12} \wedge U_{3}+U_{123} \wedge U_{13} \wedge U_{2}+U_{123} \wedge U_{23} \wedge U_{1}\right] \wedge\left(\wedge^{k-3} W^{*}\right) \\
& +U_{12} \wedge U_{13} \wedge U_{23} \wedge\left(\wedge^{k-3} W^{*}\right)+U_{123} \wedge U_{1} \wedge U_{2} \wedge U_{3} \wedge\left(\wedge^{k-4} W^{*}\right) \\
& +\left[\left(\wedge^{2} U_{12}\right) \wedge\left(\wedge^{2} U_{3}\right)+\left(\wedge^{2} U_{13}\right) \wedge\left(\wedge^{2} U_{2}\right)+\left(\wedge^{2} U_{23}\right) \wedge\left(\wedge^{2} U_{1}\right)\right] \wedge\left(\wedge^{k-4} W^{*}\right) \\
& +\left(\wedge^{2} U_{1}\right) \wedge\left(\wedge^{2} U_{2}\right) \wedge\left(\wedge^{2} U_{3}\right) \wedge\left(\wedge^{k-6} W^{*}\right) .
\end{aligned}
$$


We emphasize that with four or more subspaces analogous formulas are much more difficult, because four subspaces have moduli, see [19].

We now determine to what extent the zero sets of the skew-flattenings provide local equations for secant varieties of Grassmannians.

The first skew-flattening $\wedge^{k} W \subset W \otimes \wedge^{k-1} W$ gives rise to the subspace varieties: let

$$
S u b_{p}\left(\wedge^{k} W\right)=\left\{[z] \in \mathbb{P}\left(\wedge^{k} W\right) \mid \exists W^{\prime} \subset W, \operatorname{dim} W^{\prime}=p, z \in \wedge^{k} W^{\prime}\right\}
$$

it admits a description via a Kempf-Weyman "collapsing" of the vector bundle $\wedge^{k} \mathcal{S}_{p} \rightarrow G(p, W)$, where $\mathcal{S}_{p}$ is the tautological rank $p$ subspace bundle, i.e., the variety is the projectivization of the image of the total space of $\wedge^{k} \mathcal{S}_{p}$ in $\wedge^{k} W$. From this description one sees that $S u b_{p}\left(\wedge^{k} W\right)$ is of dimension $p(n+1-p)+\left(\begin{array}{l}p \\ k\end{array}\right)$ and its affine conormal space at a smooth point $z \in \wedge^{k} W^{\prime} \subset \wedge^{k} W$ is

$$
\hat{N}_{z}^{*} S u b_{p}\left(\wedge^{k} W\right)=\wedge^{2}\left(W^{\prime \perp}\right) \otimes \wedge^{k-2} W^{*}
$$

As a set, $S u b_{p}\left(\wedge^{k} W\right)$ is the zero locus of $\wedge^{p+1} W^{*} \otimes \wedge^{p+1}\left(\wedge^{k-1} W^{*}\right)$, the size $(p+1)$-minors of the skew-flattening. The ideal of $S u b_{p}\left(\wedge^{k} W\right)$ is simply the set of all modules $S_{\pi} W^{*} \subset S y m\left(W^{*}\right)$ such that $\ell(\pi)>p$. Its generators are known only in very few cases, see [49, §7.3].

Question. In what cases do the minors of the skew-flattenings generate the ideal of $S u b_{p}\left(\wedge^{k} W\right)$ ?

The most relevant cases for the study of secant varieties of Grassmannians are when $p=r k$, as $\sigma_{r}(G(k, W)) \subset S u b_{r k}\left(\wedge^{k} W\right)$. Note that in general $\sigma_{r}(G(k, W))$ is a much smaller subvariety than $S u b_{r k}\left(\wedge^{k} W\right)$.

Now we compute ker $\otimes$ Image $^{\perp}$ :

For $E \in G(k, W)$, write $E_{p, k-p}: \wedge^{p} W^{*} \rightarrow \wedge^{k-p} W$ for the flattening. Then

$$
\begin{aligned}
\operatorname{ker}\left(E_{2, k-2}\right) & =\wedge^{2}\left(E^{\perp}\right) \\
\operatorname{Image}\left(E_{2, k-2}\right)^{\perp} & =\left(\wedge^{k-2} E\right)^{\perp}=E^{\perp} \wedge\left(\wedge^{k-3} W^{*}\right)
\end{aligned}
$$

and similarly

$$
\begin{aligned}
\operatorname{ker}\left(\left[E_{1}+E_{2}\right]_{2, k-2}\right) & =U_{12} \wedge W^{*}+U_{1} \wedge U_{2} \\
\operatorname{Image}\left(\left[E_{1}+E_{2}\right]_{2, k-2}\right)^{\perp} & =U_{12} \wedge\left(\wedge^{k-3} W^{*}\right)+U_{1} \wedge U_{2} \wedge\left(\wedge^{k-4} W^{*}\right)
\end{aligned}
$$

And thus

$$
\begin{aligned}
& \operatorname{ker}\left(\left[E_{1}+E_{2}\right]_{2, k-2}\right) \wedge \operatorname{Image}\left(\left[E_{1}+E_{2}\right]_{2, k-2}\right)^{\perp} \\
& =\wedge^{2} U_{12} \wedge\left(\wedge^{k-2} W^{*}\right)+U_{12} \wedge U_{1} \wedge U_{2} \wedge\left(\wedge^{k-3} W^{*}\right)+\wedge^{2} U_{1} \wedge \wedge^{2} U_{2} \wedge\left(\wedge^{k-4} W^{*}\right)
\end{aligned}
$$

which agrees with (19). (Note that if we had tried the $(1, k-1)$-flattening, we would have missed the third term in (19).)

Similarly

$$
\operatorname{ker}\left(\left[E_{1}+E_{2}+E_{3}\right]_{2, k-2}\right)=U_{123} \wedge W^{*}+U_{12} \wedge U_{3}+U_{13} \wedge U_{2}+U_{23} \wedge U_{1}
$$

$$
\begin{aligned}
\text { Image }\left(\left[E_{1}+E_{2}+E_{3}\right]_{2, k-2}\right)^{\perp} & =U_{123} \wedge\left(\wedge^{k-3} W^{*}\right)+\left[U_{12} \wedge U_{3}+U_{13} \wedge U_{2}+U_{23} \wedge U_{1}\right] \wedge\left(\wedge^{k-4} W^{*}\right) \\
& +U_{1} \wedge U_{2} \wedge U_{3} \wedge\left(\wedge^{k-5} W^{*}\right)
\end{aligned}
$$

and ker $\otimes$ Image $^{\perp}$ does not contain last term in (20) if it is nonzero. However when we consider the $(3, k-3)$ flattening we will recover the full conormal space. In general, we obtain: 
Theorem 10.1.1. The variety $\sigma_{3}(G(k, W)) \subset \mathbb{P} \wedge^{k} W$ is an irreducible component of the zero set of the size $3 k+1$ minors of the skew-flattening $\wedge^{s} W \otimes \wedge^{k-s} W, s \leq k-s, k \leq \operatorname{dim} W-k$, as long as $s \geq 3$.

This theorem does not extend to $\sigma_{4}(G(k, W))$ because there it is possible to have e.g., sums of vectors in pairwise intersections of spaces that add to a vector in a triple intersection, which does not occur for the third secant variety.

Remark 10.1.2. When $\operatorname{dim} W$ is small, the range of Theorem 10.1.1 can be extended. For example, when $\operatorname{dim} W=8, \sigma_{4}(G(4,8))$ is an irreducible component of the size 9 minors of $\wedge^{2} \mathbb{C}^{8} \otimes \wedge^{6} \mathbb{C}^{8}$

We expect the situation to be similar to that of Veronese varieties, where for small secant varieties skew flattenings provide enough equations to cut out the variety, then for a larger range of $r$ the secant variety is an irreducible component of the variety of skew flattenings, and then for larger $r$ more equations will be needed.

10.2. Skew-inheritance. If $\operatorname{dim} W>m$, and $\pi$ is a partition with at most $m$ parts, we say the module $S_{\pi} W$ is inherited from the module $S_{\pi} \mathbb{C}^{m}$. The following is a straight-forward variant of [30, Prop. 4.4]:

Proposition 10.2.1. Equations (set-theoretic, scheme-theoretic or ideal theoretic) for $\sigma_{r}(G(k, W)$ ) for $\operatorname{dim} W>k r$ are given by

- the modules inherited from the ideal of $\sigma_{r}(G(k, k r))$,

- and the modules generating the ideal of $S u b_{k r}\left(\wedge^{k} W\right)$.

\section{Homogeneous varieties $G / P$}

Let $X=G / P \subset \mathbb{P} V_{\lambda}$ be a homogeneously embedded homogeneous variety, where $V_{\lambda}$ denotes the irreducible $G$-module of highest weight $\lambda$. In particular, when $\lambda=\omega_{i}$ is fundamental, $P$ is the parabolic obtained by deleting the root spaces corresponding to the negative roots $\alpha=\sum m^{j} \alpha_{j}$ such that $m^{i} \neq 0$. Let $G_{0}$ be the Levi-factor of $P$, if $W_{\mu}$ is an irreducible $\mathfrak{g}_{0}$-module given by its highest weight $\mu$, we consider it as a $\mathfrak{p}$-module by letting the nilpotent part of $\mathfrak{p}$ act trivially. We let $E_{\mu} \rightarrow G / P$ denote the corresponding irreducible homogeneous vector bundle. If $\mu$ is also $\mathfrak{g}$-dominant, then $H^{0}\left(E_{\mu}\right)^{*}=V_{\mu}$.

11.1. Example: The usual flattenings for triple Segre products. Let $A, B, C$ be vector spaces, and use $\omega, \eta, \psi$ respectively for the fundamental weights of the corresponding $S L$ modules. Then take $E$ with highest weight $\left(\omega_{1}, \eta_{1}, 0\right)$, so $E^{*} \otimes L$ has highest weight $\left(0,0, \psi_{1}\right)$. Note each of these bundles has rank one, as is the rank of $A_{v}$ when $v \in \hat{S} e g(\mathbb{P} A \times \mathbb{P} B \times \mathbb{P} C)$ as the theory predicts. We get the usual flattenings as in [28] and [18]. The equations found for the unbalanced cases in [9, Thm. 2.4] are of this form.

11.2. Example: Strassen's equations. Let $\operatorname{dim} A=3$. Taking $E$ with highest weight $\left(\omega_{2}, \eta_{1}, 0\right)$ so $E^{*} \otimes L$ has highest weight $\left(\omega_{2}, 0, \psi_{1}\right)$, yields Strassen's equations (see [39] and [29]). Each of these bundles has rank two, as is the rank of $A_{v}$ when $v \in \hat{S} e g(\mathbb{P} A \times \mathbb{P} B \times \mathbb{P} C)$.

11.3. Inheritance from usual Grassmannians. Let $\omega$ be a fundamental weight for $\mathfrak{g}$, and consider $G / P \subset \mathbb{P} V_{\omega}$. Say the Dynkin diagram of $\mathfrak{g}$ is such that $\omega$ is $s$-nodes from an end node with associated fundamental representation $V_{\eta}$. Then $V_{\omega} \subseteq \wedge^{s} V_{\eta}$ and $G / P \subset G\left(s, V_{\eta}\right)$, see, e.g., [30]. Thus $\sigma_{r}(G / P) \subset \sigma_{r}\left(G\left(s, V_{\eta}\right)\right)$, so any equations for the latter give equations for $\sigma_{r}(G / P)$. There may be several such ways to consider $\omega$, each will give (usually different) modules of equations.

For example, in the case $D_{n} / P_{n-2}, V_{\omega_{n-2}}$ is a submodule of $\wedge^{2} V_{\omega_{n}}, \wedge^{2} V_{\omega_{n-1}}$ and $\wedge^{n-2} V_{\omega_{1}}$. 
In the case of the $E$ series we have inclusions

$$
\begin{aligned}
V_{\omega_{3}} & \subset \wedge^{2} V_{\omega_{1}} \\
V_{\omega_{4}} & \subset \wedge^{2} V_{\omega_{2}} \\
V_{\omega_{n-1}} & \subset \wedge^{2} V_{\omega_{n}}
\end{aligned}
$$

Here, in each case the vector bundle $E$ has rank two, so we obtain minimal degree equations for the secant varieties of $E_{n} / P_{3}, E_{n} / P_{4}, E_{n} / P_{n-1}$. The dimension of $H^{0}(E)$ for $\left(\mathfrak{e}_{6}, \mathfrak{e}_{7}, \mathfrak{e}_{8}\right)$ is respectively: $(27,133,3875)$ for $\omega_{3},(78,912,147250)$ for $\omega_{4}$, and $(27,56,248)$ for $\omega_{n-1}$.

11.4. Other cases. From the paragraph above, it is clear the essential cases are the fundamental $G / P \subset \mathbb{P} V_{\omega}$ where $\omega$ appears at the end of a Dynkin diagram. The primary difficulty in finding equations is locating vector bundles $E$ such that $E$ and $E^{*} \otimes L$ both have sections. If $E$ corresponds to a node on the interior of a Dynkin diagram, then $E^{*}$ will have a -2 over the node of $\omega$ and $E^{*} \otimes L$ a minus one on the node, and thus no sections. However if $E$ corresponds to a different end of the diagram, one obtains nontrivial equations.

Example 11.4.1. $E_{n} / P_{\alpha_{1}} \subset \mathbb{P} V_{\omega_{1}}$ for $n=6,7,8$. Taking $E=E_{\omega_{n}}$, then $E^{*} \otimes L=E$ and $H^{0}\left(E_{\omega_{n}}\right)=V_{\omega_{n}}$. The rank of $E$ is $2(n-1)$ and the fiber is the standard representation of $S O(2 n-2)$. $\operatorname{dim} V_{\omega_{n}}$ is respectively $27,56,248$, so one gets equations for $\sigma_{r}\left(E_{n} / P_{\alpha_{1}}\right)$ for $r$ respectively up to $2,4,17$. Since the matrix is square, it is possible these equations have lower degrees than one naively expects. Especially since in the case of $E_{6}$, the secant variety is known to be a cubic hypersurface.

Example 11.4.2. $D_{n} / P_{n} \subset \mathbb{P} V_{\omega_{n}}$. Here we may take $E=E_{\omega_{1}}$ and then $E^{*} \otimes L=E_{\omega_{n-1}}$. The fiber of $E$ is the standard representation of $A_{n-1}$ in particular it is of dimension $n, H^{0}(E)=V_{\omega_{1}}$ which is of dimension $2 n$ and $H^{0}\left(E^{*} \otimes L\right)=V_{\omega_{n-1}}$. Thus these give (high degree) equations for the spinor varieties $D_{n} / P_{n}$ but no equations for their secant varieties. Some equations for $\sigma_{2}\left(D_{n} / P_{n}\right)$ are known, see $[35,33]$

\section{REFERENCES}

1. Edoardo Ballico, On the weak non-defectivity of Veronese embeddings of projective spaces, Cent. Eur. J. Math. 3 (2005), no. 2, 183-187 (electronic). MR MR2129920 (2005m:14097)

2. W. Barth, Moduli of vector bundles on the projective plane, Invent. Math. 42 (1977), 63-91. MR MR0460330 $(57 \# 324)$

3. M. Beltrametti, P. Francia, and A. J. Sommese, On Reider's method and higher order embeddings, Duke Math. J. 58 (1989), no. 2, 425-439. MR MR1016428 (90h:14021)

4. Nicolas Bourbaki, Lie groups and Lie algebras. Chapters 4-6, Elements of Mathematics (Berlin), SpringerVerlag, Berlin, 2002, Translated from the 1968 French original by Andrew Pressley. MR MR1890629 (2003a:17001)

5. David A. Buchsbaum and David Eisenbud, Algebra structures for finite free resolutions, and some structure theorems for ideals of codimension 3, Amer. J. Math. 99 (1977), no. 3, 447-485. MR MR0453723 (56 \#11983)

6. W. Buczynska and J. Buczynski, Secant varieties to high degree Veronese reembeddings, catalecticant matrices and smoothable Gorenstein schemes, arXiv:1012.3563.

7. Peter Bürgisser, Michael Clausen, and M. Amin Shokrollahi, Algebraic complexity theory, Grundlehren der Mathematischen Wissenschaften [Fundamental Principles of Mathematical Sciences], vol. 315, SpringerVerlag, Berlin, 1997, With the collaboration of Thomas Lickteig. MR 99c:68002

8. D. Cartwright, Erman D., and L. Oeding, Secant varieties of $\mathbf{P}^{2} \times \mathbf{P}^{n}$ embedded by $\mathcal{O}(1,2)$, arXiv:1009.1199, to appear in Journal L.M.S.

9. M. V. Catalisano, A. V. Geramita, and A. Gimigliano, On the ideals of secant varieties to certain rational varieties, J. Algebra 319 (2008), no. 5, 1913-1931. MR MR2392585 (2009g:14068)

10. L. Chiantini and C. Ciliberto, Weakly defective varieties, Trans. Amer. Math. Soc. 354 (2002), no. 1, 151-178 (electronic). MR MR1859030 (2003b:14063)

11. Pierre Comon, Gene Golub, Lek-Heng Lim, and Bernard Mourrain, Symmetric tensors and symmetric tensor rank, SIAM J. Matrix Anal. Appl. 30 (2008), no. 3, 1254-1279. MR MR2447451 (2009i:15039) 
12. Susan Diesel, Irreducibility and dimension theorems for families of height 3 Gorenstein algebras, Pacific J. Math 172 (1996), 365-396.

13. Lawrence Ein, Varieties with small dual varieties. I, Invent. Math. 86 (1986), no. 1, 63-74. MR $87 \mathrm{~m}: 14047$

14. David Eisenbud, Jee Koh, and Michael Stillman, Determinantal equations for curves of high degree, Amer. J. Math. 110 (1988), no. 3, 513-539. MR MR944326 (89g:14023)

15. Geir Ellingsrud and Stein Arild Stromme, Bott's formula and enumerative geometry, J. Amer. Math. Soc. 9 (1996), no. 1, 175-193. MR MR1317230 (96j:14039)

16. William Fulton, Intersection theory, second ed., Ergebnisse der Mathematik und ihrer Grenzgebiete. 3. Folge. A Series of Modern Surveys in Mathematics [Results in Mathematics and Related Areas. 3rd Series. A Series of Modern Surveys in Mathematics], vol. 2, Springer-Verlag, Berlin, 1998. MR MR1644323 (99d:14003)

17. William Fulton and Joe Harris, Representation theory, Graduate Texts in Mathematics, vol. 129, SpringerVerlag, New York, 1991, A first course, Readings in Mathematics. MR MR1153249 (93a:20069)

18. Luis David Garcia, Michael Stillman, and Bernd Sturmfels, Algebraic geometry of Bayesian networks, J. Symbolic Comput. 39 (2005), no. 3-4, 331-355. MR MR2168286 (2006g:68242)

19. I. M. Gel'fand and V. A. Ponomarev, Problems of linear algebra and classification of quadruples of subspaces in a finite-dimensional vector space, Hilbert space operators and operator algebras (Proc. Internat. Conf., Tihany, 1970), North-Holland, Amsterdam, 1972, pp. 163-237. Colloq. Math. Soc. János Bolyai, 5. MR 0357428 (50 \#9896)

20. Anthony V. Geramita, Catalecticant varieties, Commutative algebra and algebraic geometry (Ferrara), volume dedicated to M. Fiorentini, Lecture Notes in Pure and Appl. Math., vol. 206, Dekker, New York, 1999, pp. 143156.

21. A. Ginensky, A generalization of the Clifford index and determinantal equations for curves and their secant varieties, PhD. Thesis, arXiv:1002.2023.

22. Daniel R. Grayson and Michael E. Stillman, Macaulay 2, a software system for research in algebraic geometry, Available at http://www.math.uiuc.edu/Macaulay2/.

23. Joe Harris and L. Tu, On symmetric and skew-symmetric determinantal varieties, Topology 23 (1984), no. 1, 71-84.

24. D. Hilbert, Letter adresseé à M. Hermite, Gesam. Abh. vol. II 148-153.

25. Anthony Iarrobino and Vassil Kanev, Power sums, Gorenstein algebras, and determinantal loci, Lecture Notes in Mathematics, vol. 1721, Springer-Verlag, Berlin, 1999, Appendix C by Iarrobino and Steven L. Kleiman. MR MR1735271 (2001d:14056)

26. Vassil Kanev, Chordal varieties of Veronese varieties and catalecticant matrices, J. Math. Sci. (New York) 94 (1999), no. 1, 1114-1125, Algebraic geometry, 9. MR MR1703911 (2001b:14078)

27. J. M. Landsberg, Geometry and the complexity of matrix multiplication, Bull. Amer. Math. Soc. (N.S.) 45 (2008), no. 2, 247-284. MR MR2383305 (2009b:68055)

28. J. M. Landsberg and L. Manivel, On the ideals of secant varieties of Segre varieties, Found. Comput. Math. 4 (2004), no. 4, 397-422. MR MR2097214 (2005m:14101)

29. _ Generalizations of Strassen's equations for secant varieties of Segre varieties, Comm. Algebra 36 (2008), no. 2, 405-422. MR MR2387532 (2009f:14109)

30. J. M. Landsberg and Laurent Manivel, On the projective geometry of rational homogeneous varieties, Comment. Math. Helv. 78 (2003), no. 1, 65-100. MR 2004a:14050

31. _ On the ideals of secant varieties of Segre varieties, Found. Comput. Math. 4 (2004), no. 4, 397-422. MR MR2097214 (2005m:14101)

32. J.M. Landsberg, The geometry of tensors: applications to complexity, statistics and engineering, book in preparation.

33. J.M. Landsberg and J. Weyman, On secant varieties of compact hermitian symmetric spaces, J. Pure Appl. Algebra 213 (2009), no. 11, 2075-2086.

34. A. Lanteri and R. Muñoz, Discriminant loci of ample and spanned line bundles, J. Pure Appl. Algebra 212 (2008), no. 4, 808-831. MR MR2363494 (2009c:14012)

35. L. Manivel, On spinor varieties and their secants, SIGMA 5 (2009), volume spécial "Elie Cartan and Differential Geometry".

36. Massimiliano Mella, Singularities of linear systems and the Waring problem, Trans. Amer. Math. Soc. 358 (2006), no. 12, 5523-5538 (electronic). MR MR2238925 (2007h:14059)

37. David Mumford, Lectures on curves on an algebraic surface, With a section by G. M. Bergman. Annals of Mathematics Studies, No. 59, Princeton University Press, Princeton, N.J., 1966. MR MR0209285 (35 \#187)

38. L. Oeding and G. Ottaviani, Eigenvectors of tensors and algorithms for Waring decomposition, arXiv:1103.0203. 
39. Giorgio Ottaviani, Symplectic bundles on the plane, secant varieties and Lüroth quartics revisited, Vector Bundles and Low Codimensional Subvarieties: State of the Art and Recent Developments (R. Notari G. Casnati, F. Catanese, ed.), Quaderni di Matematica, vol. 21, Dip. di Mat., II Univ. Napoli, 2007, pp. 315-352.

40. __ An invariant regarding Waring's problem for cubic polynomials, Nagoya Math. J. 193 (2009), 95-110.

41. Lior Pachter and Bernd Sturmfels (eds.), Algebraic statistics for computational biology, Cambridge University Press, New York, 2005. MR MR2205865 (2006i:92002)

42. F. Palatini, Sulla rappresentazione delle forme ternarie mediante la somma di potenze di forme lineari, Rom. Acc. L. Rend. 12 (1903), 378-384.

43. C. Raicu, $3 \times 3$ minors of Catalecticants, arXiv:1011.1564.

44. H.W. Richmond, On canonical forms, Quart. J. Pure Appl. Math. 33 (1904), 967-984.

45. Steven V. Sam, Computing inclusions of Schur modules, The J. of Software for Alg. and Geom. 1 (2009), $5-10$.

46. Frank-Olaf Schreyer, Geometry and algebra of prime Fano 3-folds of genus 12, Compositio Math. 127 (2001), no. 3, 297-319. MR MR1845040 (2002d:14062)

47. Richard P. Stanley, Hilbert functions of graded algebras, Advances in Math. 28 (1978), no. 1, 57-83. MR MR0485835 (58 \#5637)

48. V. Strassen, Rank and optimal computation of generic tensors, Linear Algebra Appl. 52/53 (1983), 645-685. MR 85b:15039

49. Jerzy Weyman, Cohomology of vector bundles and syzygies, Cambridge Tracts in Mathematics, vol. 149, Cambridge University Press, Cambridge, 2003. MR MR1988690 (2004d:13020)

E-mail address: jml@math.tamu.edu, ottavian@math.unifi.it 